\title{
Article \\ Elucidation of the Natural Function of Sophorolipids Produced by Starmerella bombicola
}

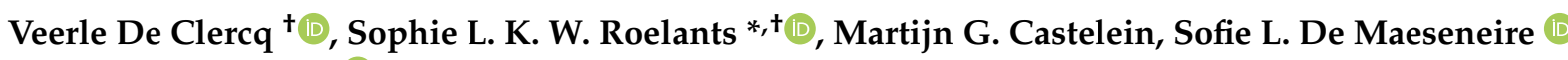 \\ and Wim K. Soetaert (D)
}

Citation: De Clercq, V.; Roelants, S.L.K.W.; Castelein, M.G.; De

Maeseneire, S.L.; Soetaert, W.K.

Elucidation of the Natural Function of Sophorolipids Produced by Starmerella bombicola. J. Fungi 2021, 7, 917. https://doi.org/10.3390/ jof7110917

Academic Editor: Aaron Neiman

Received: 5 October 2021

Accepted: 22 October 2021

Published: 28 October 2021

Publisher's Note: MDPI stays neutral with regard to jurisdictional claims in published maps and institutional affiliations.

Copyright: (c) 2021 by the authors. Licensee MDPI, Basel, Switzerland. This article is an open access article distributed under the terms and conditions of the Creative Commons Attribution (CC BY) license (https:// creativecommons.org/licenses/by/ $4.0 /)$.
Centre for Industrial Biotechnology and Biocatalysis (InBio.be), Department of Biotechnology, Faculty of Bioscience Engineering, Ghent University, Coupure Links 653, 9000 Ghent, Belgium; veedcler.DeClercq@UGent.be (V.D.C.); Martijn.Castelein@UGent.be (M.G.C.);

Sofie.DeMaeseneire@UGent.be (S.L.D.M.); Wim.Soetaert@UGent.be (W.K.S.)

* Correspondence: Sophie.Roelants@UGent.be; Tel.: +32-9-264-93-85

+ These authors contributed equally to this work.

\begin{abstract}
The yeast Starmerella bombicola distinguishes itself from other yeasts by its potential of producing copious amounts of the secondary metabolites sophorolipids (SLs): these are glycolipid biosurfactants composed out of a(n) (acetylated) sophorose moiety and a lipid tail. Although SLs are the subject of numerous research papers and have been commercialized, e.g., in eco-friendly cleaning solutions, the natural function of SLs still remains elusive. This research article investigates several hypotheses for why S. bombicola invests that much energy in the production of SLs, and we conclude that the main natural function of SLs in S. bombicola is niche protection: (1) the extracellular storage of an energy-rich, yet metabolically less accessible carbon source that can be utilized by S. bombicola upon conditions of starvation with (2) antimicrobial properties. In this way, S. bombicola creates a dual advantage in competition with other microorganisms. Additionally, SLs can expedite growth on rapeseed oil, composed of triacylglycerols which are hydrophobic substrates present in the yeasts environment, for a non-SL producing strain ( $\Delta$ cyp52M1). It was also found that-at least under lab conditions-SLs do not provide protection against high osmotic pressure prevalent in sugar-rich environments such as honey or nectar present in the natural habitat of S. bombicola.
\end{abstract}

Keywords: Starmerella bombicola; biosurfactants; sophorolipids; physiological function; natural role; antimicrobial; exclusive storage compound

\section{Introduction}

The name 'Starmerella bombicola' strongly associates with 'biosurfactants' (bio-based surface-active agents), as this species is the best known producer of sophorolipids, which are secondary metabolites: more than $75 \%$ of the research publications on this yeast focus on sophorolipids [1,2]. Sophorolipids (SLs) are amphiphilic molecules with a hydrophilic sophorose head, consisting of two $\beta-1,2$ linked glucose molecules, and a hydrophobic fatty acid tail. The SLs produced by S. bombicola are typically a mixture of several slightly different congeners providing each of them with specific properties [3-8]. For example, SL molecules with a free carboxylic group are called acidic SLs and have good solubility in water. This carboxyl group can be esterified with the hydroxyl group on the C4" of sophorose forming a macrocyclic ring giving rise to lactonic SLs, which are characterized by low water solubility. Examples of other diversifications are: the length and saturation degree of the fatty acid chain (for S. bombicola, mainly C16:1 or C18:1), the position of attachment of the sophorose moiety (through a glycosidic bond) to the fatty acid at the $\omega$ (terminal) or $\omega-1$ (sub-terminal) position, and the acetylation degree of sophorose (major structures are shown in Figure 1) $[3,9,10]$.

The 'soap-like' structure of SLs provides them with wetting, emulsification, foaming, and dispersing properties combined with a sustainable and environmental-friendly char- 
acter. These properties make them attractive for the detergent industry, medical world, personal care sector, mining processes, food industry, cosmetics, crop protection, pharmaceuticals, and bio-remediation [11-17]. This broad industrial relevance attracts the attention of many researchers, resulting in ample and diverse application investigations [2]. On the other hand, several laboratory tools were developed over the past decade, gradually turning S. bombicola into an engineerable organism and, as such, expanding the portfolio of molecular structures and concomitant functions and applications [16,18-21]. Although the research on S. bombicola and its produced molecules has given rise to an exponential growth of research papers on the subject, the natural function of sophorolipids in Starmerella bombicola still remains obscure [1].

a

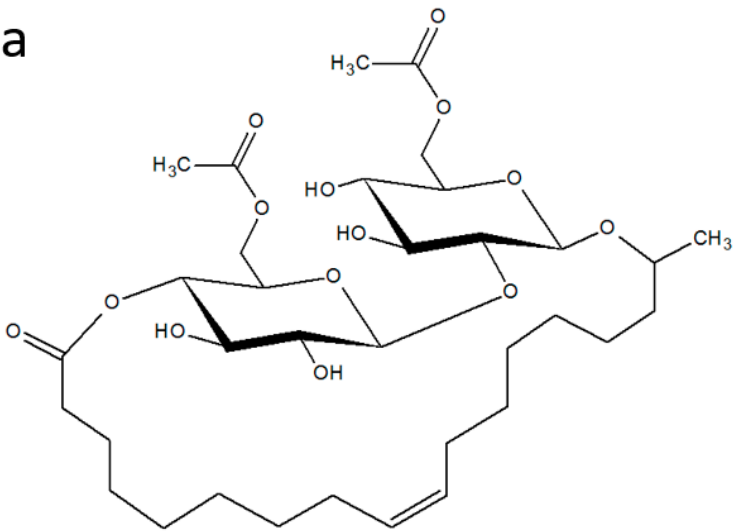

b

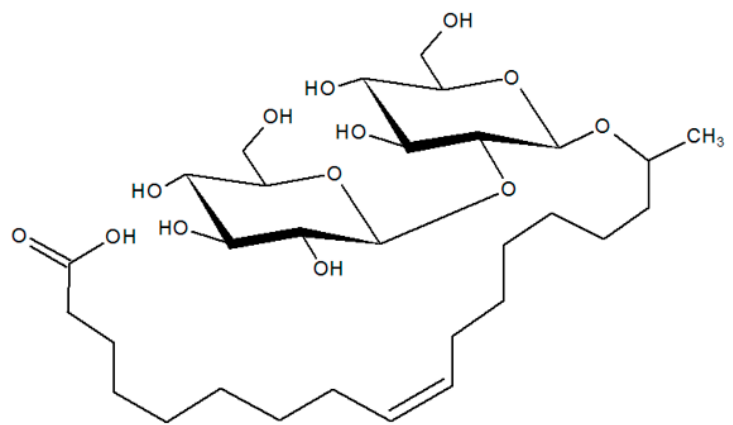

Figure 1. The structure of a lactonic (a) and an acidic (b) sophorolipid (SL), produced by (amongst others) Starmerella bombicola. Both glucose molecules can be acetylated on position C6 as shown in (a); these acetyl groups can be completely absent as shown in (b), but also mono-acetylated SLs are produced.

The aim of this study is to resolve this fascinating microbial mystery in a rational manner; the answer to the question 'why does S. bombicola produce SLs?' should be related to improved fitness, yielding in an immediate or delayed growth benefit. The fact that SLs are secondary metabolites indicates they do not fulfill (an) essential function(s) in cell growth and/or maintenance, but their presence favors the producing organism in specific conditions. A first aspect that needs to be considered is the natural environment of S. bombicola and its associated characteristics. S. bombicola was isolated from nectar samples of wild flowers (in 1954) and bumblebee honey (1964-1967) by Spencer et al. [22]. They identified this new yeast species as Torulopsis bombicola due to its close association with bumblebees. Later on, the yeast was reclassified as Candida bombicola and finally renamed Starmerella bombicola in 2012 [23,24]. In the years following the discovery, additional S. bombicola strains were isolated all over the world, primarily originating from samples derived from flowers and (bumble)bees (and exceptionally also from some other flower visiting insects) [2]. The associated physical and biological factors characterizing these habitats and affecting the microorganisms colonizing it are mainly: high osmotic pressure (low water activity) due to the abundance of sugars in honey (and in lesser concentrations also in nectar) (see Table 1), floral microhabitats with dynamic microbial communities due to high dispersal and foraging by (pollinating) visitors, and the availability of a wide variety of substrates including hydrophobic substrates—such as (mono)esters of long chain fatty acids in beeswax and triacylglycerols in plant oils of flower seeds [25-29].

Several plausible hypotheses on the natural role of SLs in Starmerella bombicola have been postulated in the literature and can be summarized as follows: (1) SL production constitutes an overflow metabolism [30]; (2) SLs exert antimicrobial activity, thereby inhibiting the growth of competing microorganisms [15,31-37]; (3) SL production is a protection mechanism against high osmotic pressure [38]; (4) SLs improve the uptake of hydrophobic substrates [39,40]; and (5) SLs serve as an extracellular storage compound of carbon and energy [38]. 
Table 1. Sugar concentrations $(\% w / w)$ in honey of honey bees (Apis mellifera) and bumblebees (Bombus sp.) and in nectar of Berberis darwinii [25,26].

\begin{tabular}{cccc}
\hline$\%$ w/w & Honey of A. mellifera & Honey of Bombus sp. & Nectar of B. darwinii \\
\hline Sucrose & $1-3$ & $0.6-3$ & 33.1 \\
Glucose & $30-48$ & $5-54$ & 1.2 \\
Fructose & $41-54$ & $37-79$ & 0.6 \\
Water (and other) & $13.4-26.6$ & $13-30$ & 63.7 \\
\hline
\end{tabular}

The first hypothesis (1) that SL production constitutes an overflow metabolism in order to regulate intracellular energy levels was proposed by Davila et al. [30]. This theory can be easily rejected based on convenient knowledge: overflow metabolism relates to a deficit in the cofactor $\mathrm{NAD}^{+}$, which cannot be regenerated through the biosynthesis of SLs [41]; an elaborated argumentation can be found in Dierickx et al. [42]. The latter three hypotheses (3-5) are rather speculative without substantiated data and are therefore investigated in this research article, together with the hypothesis on the antimicrobial activity (2) for which some preliminary data exist. This study is the first one to answer the question 'Why does S. bombicola produce SLs?' in a more thorough way. According to the findings, SL production is not accompanied by elevated protection against high osmotic pressure arising from high sugar concentrations. It was found however that S. bombicola can catabolize its previously synthesized SLs in times of starvation, and the antimicrobial activity was confirmed. We conclude that the main natural function of SLs for S. bombicola can be defined as niche protection through a dual advantage: the build-up of an exclusive and extracellular storage compound, characterized by antimicrobial properties. As an additional advantage, S. bombicola could possibly benefit from SLs in the presence of hydrophobic substrates (e.g., triacylglycerols), as improved growth rates were noticed on rapeseed oil for the SL deficient $\Delta c y p 52 M 1$ strain by adding SLs (but an adverse effect was found for the alkane hexadecane).

\section{Materials and Methods}

\subsection{In Vitro Antimicrobial Assay}

For the antimicrobial activity of SLs, the following wild-type laboratory strains were chosen: Gram-positive bacteria (Bacillus subtilis LMG 2099, Staphylococcus aureus LMG 8224, Fructobacillus fructosus LMG 30235), Gram-negative bacteria (Escherichia coli MG 1655, Pseudomonas aeruginosa LMG 24986, Hafnia alvei LMG 28933), and yeast species (Candida albicans SC 5314, Zygosaccharomyces rouxii MUCL 30008, Starmerella bombicola ATCC 22214). Precultures of E. coli, P. aeruginosa, and B. subtilis were grown on Lysogeny broth (10 g. $\mathrm{L}^{-1}$ tryptone, $5 \mathrm{~g} \cdot \mathrm{L}^{-1}$ yeast extract and $\left.5 \mathrm{~g} \cdot \mathrm{L}^{-1} \mathrm{NaCl}\right) ; \mathrm{H}$. alvei was cultivated on nutrient broth (Oxoid); S. aureus was cultivated on brain heart infusion broth (Biokar diagnostics); F. fructosus was cultivated on MRS broth (Thermo Fisher Scientific); C. albicans and S. bombicola were cultivated on $3 \mathrm{C}$ medium $\left(100 \mathrm{~g} \cdot \mathrm{L}^{-1}\right.$ glucose, $10 \mathrm{~g} \cdot \mathrm{L}^{-1}$ yeast extract, and $1 \mathrm{~g} \cdot \mathrm{L}^{-1}$ urea), and $Z$. rouxii was cultivated on YPD medium $\left(5 \mathrm{~g} \cdot \mathrm{L}^{-1}\right.$ yeast extract, $10 \mathrm{~g} \cdot \mathrm{L}^{-1}$ peptone and $20 \mathrm{~g} \cdot \mathrm{L}^{-1}$ glucose). All bacterial precultures, except for $F$. fructosus and $H$. alvei, were cultivated at $37^{\circ} \mathrm{C}$ and $200 \mathrm{rpm}$ for 24 to $36 \mathrm{~h}$. H. alvei, F. fructosus, and the yeast strains were grown at $30^{\circ} \mathrm{C}$ and $200 \mathrm{rpm}$ for 48 to $72 \mathrm{~h}$.

For the antimicrobial assay, Mueller Hinton broth (MHB, Biokar diagnostics) was used at a $\mathrm{pH}$ of 4 for yeasts and a $\mathrm{pH}$ of 7 for bacteria using a citrate-phosphate buffer (Mcllvaine buffer, 0.1 to $0.2 \mathrm{M}$ depending on the $\mathrm{pH}$ setpoint). Precultures were diluted in $\mathrm{MHB}$ to a turbidity of the $0.5 \mathrm{McFarland}$ standard, which equals approximately $1-2 \times 10^{8} \mathrm{CFU} \cdot \mathrm{mL}^{-1}$, and were subsequently inoculated at $1.3 \%$ in 96-well microtiter plates (MTP) (Greiner Bio-One) with a total volume of $150 \mu \mathrm{L}$ MHB with varying SL concentrations ranging from $0.5 \mathrm{~g} \cdot \mathrm{L}^{-1}$ up to 20 or $30 \mathrm{~g} \cdot \mathrm{L}^{-1}$. These MTPs were incubated for $24 \mathrm{~h}$ on a MTP shaker (700 rpm) at $30^{\circ} \mathrm{C}$ (yeasts) or $37^{\circ} \mathrm{C}$ (bacteria). Due to growth difficulties on MHB for Z. rouxii and $F$. fructosus, aberrant media/conditions were used; these strains were cultivated on a YPD or MRS medium, respectively, at $30^{\circ} \mathrm{C}$ for $48 \mathrm{~h}$. 
Three different C18:1/C18:0 sophorolipid (SL) compounds (purity 99\%) were evaluated for their antimicrobial activity (prepared as described below in 2.3): non-acetylated acidic SLs (uniformity 99.7\%), di-acetylated lactonic SL (uniformity 95.0\%), and a wild-type SL mixture. SLs were prepared as $200 \mathrm{~g} \cdot \mathrm{L}^{-1}$ (acidic and lactonic SLs) or $300 \mathrm{~g} \cdot \mathrm{L}^{-1}$ (wildtype SL mixture) stock solutions in dimethyl sulfoxide (DMSO) to circumvent solubility issues. Therefore, a preliminary DMSO serial dilution test (up to $10 \% v / v$ ) was used to evaluate microbial viability upon the DMSO addition. The maximum tested SL concentration per microbial strain depended on the highest DMSO concentration that did not affect growth of that strain, with a maximum SL concentration of $20 \mathrm{~g} \cdot \mathrm{L}^{-1}$ (acidic and lactonic SLs) or $30 \mathrm{~g} \cdot \mathrm{L}^{-1}$ (wild-type SL mixture), corresponding to a DMSO concentration of $10 \%$. Per strain, the determined maximum DMSO concentration that should not affect growth was included in parallel in the assay as a control.

Minimum inhibitory concentration (MIC) and minimum lethal concentration (MLC) values were determined in duplicate $(n=2)$ using a 96-well plate serial dilution method based on Clinical and Laboratory Standards Institute (CLSI) guidelines [43-45]. A two-fold serial dilution of SLs ranging from $\sim 0.5 \mathrm{~g} \cdot \mathrm{L}^{-1}$ up to 20 or $30 \mathrm{~g} \cdot \mathrm{L}^{-1}$ (or lower in the case of DMSO sensitive organisms) was made by mixing the SL stock solution (20 or $30 \mathrm{~g} \cdot \mathrm{L}^{-1}$ ) with the testing medium (MHB, MRS, or YPD) with a total volume of $148 \mu \mathrm{L}$ and was subsequently inoculated (total volume of $150 \mu \mathrm{L}$ ). During cultivation ( 24 or $48 \mathrm{~h}$ ), growth was monitored by optical density measurements at $600 \mathrm{~nm}$ every $7 \mathrm{~h}$ (FLUOstar OPTIMA FL, BMG Labtech). As (lactonic) SLs interfere with $\mathrm{OD}_{600}$ measurements, growth was also evaluated using a resazurin assay [46]. This fluorometric/colorimetric assay is based on the reduction of the blue dye resazurin (absorbance at $600 \mathrm{~nm}$ ) with a formation of the red fluorescent dye resofurin (ex/em of 530-560 nm/590 nm) by metabolic active cells. In practice, $30 \mu \mathrm{L}$ of a $0.2 \mathrm{~g} \cdot \mathrm{L}^{-1}$ filter sterilized resazurin solution (Sigma-Aldrich) was added to the cell cultures in the MTP after 24 or $48 \mathrm{~h}$ of cultivation and incubated for 2 extra hours. Microbial growth was verified through fluorescence measurements of resorufin at $560 \mathrm{~nm} / 590 \mathrm{~nm}$ (FLUOstar OPTIMA FL, BMG Labtech). MIC values were assigned as the lowest concentration of SLs inhibiting growth of the microorganisms (evaluated through $\mathrm{OD}_{600}$ and fluorescence measurements), while MLC values were determined as the lowest SL concentration at which no microbial growth was observed anymore after inoculation into the fresh medium (MHB, MRS, or YPD).

\subsection{Strains, Media, and Culture Conditions}

In this study, the wild-type strain Starmerella bombicola ATCC 22214, a S. bombicola $\Delta c y p 52 M 1$ strain deficient in the first step of SL biosynthesis [47], and a S. bombicola $\Delta$ sble strain $[48,49]$ were used. Yeast cells were maintained on the YPD medium $\left(10 \mathrm{~g} \cdot \mathrm{L}^{-1}\right.$ yeast extract, $20 \mathrm{~g} \cdot \mathrm{L}^{-1}$ peptone, and $20 \mathrm{~g} \cdot \mathrm{L}^{-1}$ glucose $)$ or $3 \mathrm{C}$ medium $\left(100 \mathrm{~g} \cdot \mathrm{L}^{-1}\right.$ glucose, $10 \mathrm{~g} \cdot \mathrm{L}^{-1}$ yeast extract, and $1 \mathrm{~g} \cdot \mathrm{L}^{-1}$ urea) with the addition of $20 \mathrm{~g} \cdot \mathrm{L}^{-1}$ agar (Biokar diagnostics) when required.

Shake flask experiments for the production of sophorolipids (SLs) $\left(30^{\circ} \mathrm{C}\right.$ and $\left.200 \mathrm{rpm}\right)$ were performed with the wild-type Starmerella bombicola ATCC 22214 strain in the production medium described by Lang et al. (see 'production medium' in Table 2) to which 3.75\% rapeseed oil (from a local supermarket) (or 3.75\% oleic acid when specified, Sigma-Aldrich) was added after $48 \mathrm{~h}$ [50]. The cultivation was stopped after 2 weeks (after confirmation that all of the hydrophobic substrate was consumed). The SLs were purified from these cultures as described below.

The growth experiments to investigate the effect of high osmotic pressure were executed in 96-well microtiter plates (MTP) (Greiner Bio-One) with $200 \mu \mathrm{L}$ of the SD CSM medium (Synthetic Defined medium with Complete Supplement Mixture) supplemented with varying glucose or fructose concentrations (ranging from 20 to $600 \mathrm{~g} \cdot \mathrm{L}^{-1}$ ). The composition of the SD CSM medium with $20 \mathrm{~g} \cdot \mathrm{L}^{-1}$ glucose can be found in Table 2. All components were filter sterilized separately. For preculture preparation, six single colonies of each strain $(n=6)$ were transferred from YPD plates into $200 \mu \mathrm{L}$ of the SD CSM medium 
supplied with $100 \mathrm{~g} \cdot \mathrm{L}^{-1}$ glucose or fructose, incubated at $30^{\circ} \mathrm{C}$ for 2 days on a MTP shaker (800 rpm) and subsequently used to inoculate the final MTP at 1\% (total well volume $200 \mu \mathrm{L}$ ). The final plate was incubated in an Infinite 200 PRO multimode reader (Tecan) for $60 \mathrm{~h}$ at $30{ }^{\circ} \mathrm{C}$ and orbital shaking with an amplitude of $2 \mathrm{~mm}$.

Table 2. Composition of the used media.

\begin{tabular}{|c|c|c|c|c|}
\hline Component $\left(\mathrm{g} \cdot \mathrm{L}^{-1}\right)$ & $\begin{array}{l}\text { Production } \\
\text { Medium 1,a }\end{array}$ & SL Medium ${ }^{1, b}$ & $\begin{array}{c}\text { SD CSM } \\
\text { Medium }^{2}\end{array}$ & $\begin{array}{c}\text { SD CSM } \\
\text { Medium w/o } \\
\text { C-source }^{3}\end{array}$ \\
\hline Glucose (Cargill) & 120 & / & 20 & / \\
\hline YNB $w / o$ AA (BD Difco) & / & 4 & 6.7 & 6.7 \\
\hline CSM (MP biomedicals) & / & / & 0.79 & 0.79 \\
\hline Yeast extract (Brenntag) & 4 & / & / & / \\
\hline $\begin{array}{c}\text { 3Na-citraat. } 2 \mathrm{H}_{2} \mathrm{O} \\
\text { (Sigma-Aldrich) }\end{array}$ & 5 & / & / & / \\
\hline $\mathrm{NH}_{4} \mathrm{Cl}$ (Sigma-Aldrich) & 1.5 & 1.5 & / & / \\
\hline $\mathrm{KH}_{2} \mathrm{PO}_{4}$ (Sigma-Aldrich) & 1 & 1 & / & / \\
\hline $\mathrm{K}_{2} \mathrm{HPO}_{4}$ (Sigma-Aldrich) & 0.16 & 0.16 & / & / \\
\hline $\begin{array}{c}\mathrm{MgSO}_{4} \cdot 7 \mathrm{H}_{2} \mathrm{O} \\
\text { (Sigma-Aldrich) }\end{array}$ & 0.7 & 0.7 & / & / \\
\hline $\mathrm{NaCl}$ (Esco) & 0.5 & 0.5 & / & / \\
\hline $\mathrm{CaCl}_{2} \cdot 2 \mathrm{H}_{2} \mathrm{O}$ (Merck) & 0.27 & 0.27 & / & / \\
\hline Sophorolipids & / & 20 & / & / \\
\hline
\end{tabular}

Used for the experiments in result section: ${ }^{1}$ Section 3.4. Exclusive storage compound: ${ }^{\text {a }}$ Section 3.4.1. Growth on production medium; and ${ }^{\mathrm{b}}$ Section 3.4.2. Growth on sophorolipids as the sole carbon source. ${ }^{2}$ Section 3.2. Protection against osmotic pressure. ${ }^{3}$ Section 3.3. Uptake of hydrophobic substrates.

To examine growth on hydrophobic substrates, precultures were prepared from three single colonies on YPD plates in test tubes containing $5 \mathrm{~mL}$ of the SD CSM medium supplemented with only $2 \mathrm{~g} \cdot \mathrm{L}^{-1}$ of glucose (instead of $20 \mathrm{~g} \cdot \mathrm{L}^{-1}$ ) to avoid carry over of glucose to the final experiment. A synthetic defined medium was used to minimize growth on other medium compounds, which was confirmed by a lack of growth in the negative controls (SD CSM medium without C-source) for at least 7 days. After 3 days of growth, $1 \mathrm{~mL}$ of the preculture was centrifuged; the cell pellets were washed (to avoid potential carry over of the remaining glucose or produced SLs, confirmed with TLC) and subsequently resolved with a sterile physiological solution. The volumes of the physiological solution were chosen such to obtain equal final cell concentrations (based on $\mathrm{OD}_{600}$ values). Finally, the cells were inoculated at $3 \%$ into test tubes containing $5 \mathrm{~mL}$ of the SD CSM medium (Table 2) supplemented with $20 \mathrm{~g} \cdot \mathrm{L}^{-1}$ of three different hydrophobic carbon sources as the sole C-source (in duplicate or triplicate): beeswax (Weyn's honing; $n=2$ ), hexadecane (Sigma-Aldrich, $n=3$ ), or rapeseed oil (from a local supermarket, $n=3$ ). Positive and negative controls consisted of the SD CSM medium with $20 \mathrm{~g} \cdot \mathrm{L}^{-1}$ glucose and the SD CSM medium without any C-source (no glucose), respectively. All components were autoclaved separately. A sterile wild-type sophorolipid mixture (prepared as described below) was added to one of two replicas of the same colony in a concentration of $1 \mathrm{~g} \cdot \mathrm{L}^{-1}$. The cultures were incubated for 14 days at $30^{\circ} \mathrm{C}$ and $200 \mathrm{rpm}$.

An adapted medium was used when growth on SLs (di-acetylated lactonic) as the sole C-source was envisaged. This growth medium will be called the SL medium and can be found in Table 2. The SLs (produced as described below) were added to the medium components, and the mixtures were subsequently filter sterilized (Corning). Due to growth difficulties and to prepare the cells to metabolize SLs, an alternative method was used to start up precultures for the culture on the SL medium: $5 \mathrm{~mL}$ of an old culture on the production medium (cultivated for $>20$ days) were spun down, and the harvested cells were dissolved in $5 \mathrm{~mL}$ of the SL medium. After incubation at $30^{\circ} \mathrm{C}$ and $200 \mathrm{rpm}$ for at least $24 \mathrm{~h}$ (until microscopic observations showed that cells were clearly budding), this culture was used as a second inoculum to prepare a second preculture on the SL 
medium (5 mL), which was in turn used as an inoculum for a shake flask experiment on the SL medium ( $200 \mathrm{~mL}, n=3)$. For the experiment investigating SL degradation during prolonged cultivation on the production medium, three $1 \mathrm{~L}$ shake flasks with $200 \mathrm{~mL}$ of the medium were inoculated (2\%) with overnight grown precultures, prepared from three single colonies $(n=3)$ on a $3 \mathrm{C}$ plate in $5 \mathrm{~mL}$ of the production medium.

\subsection{Preparation of (Partly) Purified Sophorolipids}

Sophorolipids (SLs) were produced by the wild-type strain Starmerella bombicola ATCC 22214 in the production medium (with the addition of rapeseed oil, see above). Analytics were performed as described in Section 2.7.

Di-acetylated lactonic SLs (C18:1) were prepared in a very pure (purity 99\%) white powder (uniformity 95.0\%) via crystallization, and non-acetylated acidic SLs (C18:1) (uniformity $99.7 \%$ ) were derived from lactonic SLs through alkaline hydrolysis and purified as described by Roelants et al. [49].

To produce the wild-type SL mixture, used for the hydrophobic substrate experiment and the in vitro antimicrobial assay, oleic acid was added as the hydrophobic substrate. The wild-type SL mixture was partly purified through melting as described by Roelants et al. [49]: the production broth was heated to $70{ }^{\circ} \mathrm{C}$ in a separation funnel, and the melted SLs collected at the bottom. SLs were tapped off and thoroughly washed with $\mathrm{dH}_{2} \mathrm{O}$. This process was repeated with the washed SL mixture. The final composition consisted of 88\% C18:1 di-acetylated lactonic SLs, 2\% C18:0 di-acetylated lactonic SLs, 7\% C18:1 mono-acetylated lactonic SLs, and 3\% C18:1 di-acetylated acidic SL (based on the peak area from ELSD); there were no leftover traces of the oleic acid substrate.

\subsection{Sampling and Determination of Growth Parameters}

The effect of high osmotic pressure was investigated with continuous $\mathrm{OD}_{600}$ measurements in an Infinite 200 PRO multimode reader (Tecan) and was based on six replicates $(n=6)$. The growth parameters were determined in $\mathrm{R}(4.0 .2)$ by using smoothing splines (for maximal growth rate $\mu_{\max }$ ) and the Gompertz function (for the determination of the lag phase and $\triangle \mathrm{OD}$ ) from the 'growthrates' package (https://cran.r-project.org/web/ packages/growthrates/vignettes/Introduction.html, accessed on 28 December 2019). As some of the sub-datasets were not normally distributed, the Mann-Whitney U test was used for statistical analysis.

For the growth experiment on hydrophobic substrates, the test tubes (duplicates for beeswax and triplicates for rapeseed oil and hexadecane) were sampled at regular time points (see Figure S5 for the timings): $75 \mu \mathrm{L}$ of the broth were mixed with $75 \mu \mathrm{L}$ of the physiological solution, and the $\mathrm{OD}_{600}$ of the solution was determined using a microplate reader (FLUOstar OPTIMA FL, BMG Labtech). The measured $\mathrm{OD}_{600}$ values were first corrected for the average optical density that was caused by the addition of sophorolipids to each substrate (so the average of the differences in OD values at $t=0$ for each replica/colony for that substrate). Three growth parameters were estimated from the obtained log transformed growth curves: an approximately estimated growth rate $\mu$, the estimated duration of the lag phase, and the $\Delta \mathrm{OD}$ (which equals the grown number of cells). Due to the discontinuous sampling/measuring, the data were not fitted for the 'growthrates' package (see above). Therefore, smoothing splines were used in $R$ (4.0.2) to estimate $\mu$ (the maximum first derivative to the smoothed spline) and the duration of the lag phase (the intersection of that estimated maximal slope line with the horizontal $\mathrm{y}=\mathrm{x}_{0}$ ). The parameters 'estimated $\mu^{\prime}$ and 'lag' of the growth curves that did not exceed the maximum corrected OD value of the negative controls (which are non-inoculated SD CSM media with beeswax, hexadecane, or rapeseed oil; and inoculated SD CSM media without the C-source, all with and without the addition of SLs) were set at $\mu=0$ and lag = Inf, respectively. For the sub-dataset with rapeseed oil as the substrate $(n=3)$, a paired t-test was used for the statistical analysis.

In the SL degradation experiments, three shake flask cultures $(n=3)$ were sampled regularly for cell concentration (CFU), glucose concentration, and $\mathrm{pH}$ determination; a su- 
pernatant collection was performed for the ammonium concentration and SL composition (see below). Glucose concentrations were determined using the 2700 select biochemistry analyzer (YSI Inc.); samples were diluted below $7.5 \mathrm{~g} \cdot \mathrm{L}^{-1}$ with $\mathrm{dH}_{2} \mathrm{O}$. The amount of free ammonium $\left(\mathrm{NH}_{4}{ }^{+}\right.$-ions) in the cell free culture medium was determined using the Ammonia Rapid Megazyme Kit. A standard solution was prepared using $\mathrm{NH}_{4} \mathrm{Cl}$. The viability of yeast cells in prolonged cultivation experiments was assessed by determining colony forming units (CFU) on $3 \mathrm{C}$ agar plates (incubation at $30^{\circ} \mathrm{C}$ for three days).

\subsection{Assays with Extracellular Secretomes}

Unconcentrated secretomes, i.e., cell free culture media containing all the extracellular proteins, were prepared in triplicate $(n=3)$ from $S$. bombicola cultures cultivated on the production medium for 21 days (see strains, media, and culture conditions). Centrifugation (15 min, $4000 \times g, 4^{\circ} \mathrm{C}$ ) of the culture broth was followed by an additional centrifugation step to remove all insolubles. The resulting cleared supernatant $(200 \mathrm{~mL})$ was divided into three volumes: the first volume was supplemented with di-acetylated lactonic SLs $\left(20 \mathrm{~g} \cdot \mathrm{L}^{-1}\right)$, the second volume with non-acetylated acidic SLs $\left(20 \mathrm{~g} \cdot \mathrm{L}^{-1}\right)$, and the last one with an equal amount of sterile $\mathrm{dH}_{2} \mathrm{O}$ to control for the background of remaining SLs and/or other compounds in the protein solutions. The resulting solutions were filter sterilized using a $0.22 \mu \mathrm{m}$ filter (Corning) and incubated $\left(30^{\circ} \mathrm{C}\right)$ in sterile shake flasks on a rotary shaker $(200 \mathrm{rpm})$. Two flasks with the fresh production medium without glucose and set at the $\mathrm{pH}$ of the collected secretomes $(\mathrm{pH}=5.5)$ were supplemented with both forms of SLs, respectively, and served as controls. The flasks were incubated for one month and sampled regularly for SL analysis, glucose, and $\mathrm{pH}$ determination.

For concentrated secretomes, three tablets of the cOmplete protease inhibitor cocktail (Roche) were added to $200 \mathrm{~mL}$ of the cleared supernatant (see above, $n=3$ ), and the secretomes were subsequently filtered using a $0.22 \mu \mathrm{m}$ filter. Secretomes were subsequently concentrated using a stirring ultra-filtration cell (Model 8200 Milipore) containing a $10 \mathrm{kDa}$ cut off $\mathrm{G}$ membrane (Sartorius Stedim). After concentrating to $30 \mathrm{~mL}$, one volume of wash buffer (20 mM Tris, pH 7) was added. The secretome was subsequently concentrated again to $30 \mathrm{~mL}$. The protein concentration was determined using a BCA Protein Assay Kit (Pierce). In case the concentration was lower than $1 \mathrm{mg} \cdot \mathrm{mL}^{-1}$, the secretomes were further concentrated using a Vivaspin 15R (Sartorius stedim). The obtained concentrated secretomes were divided into $2 \mathrm{~mL}$ Eppendorf tubes $\left(1 \mathrm{~mL}\right.$ at $1 \mathrm{mg} \cdot \mathrm{mL}^{-1}$ protein) and supplemented with $0.5 \mathrm{mM} \mathrm{MgCl} 2$ and $2 \mathrm{mM}$ of di-acetylated lactonic SLs $\left(1.4 \mathrm{~g} \cdot \mathrm{L}^{-1}\right)$ or non-acetylated acidic SLs $\left(1.2 \mathrm{~g} \cdot \mathrm{L}^{-1}\right)$. Two controls were included: a first control consisted of $2 \mathrm{mM}$ of SLs in the wash buffer to check for a potential spontaneous breakdown of SLs. A second one consisted of the concentrated secretome preparations supplemented with $\mathrm{dH} 20$ (instead of SLs) to correct for the background of remaining SLs and/or other compounds in the protein solutions. All assays were incubated for 14 days $\left(30^{\circ} \mathrm{C}\right.$, on a rotary wheel at $30 \mathrm{rpm})$.

\subsection{Assays with Intracellular Cell Lysates}

Cell pellets of $S$. bombicola cultures $(n=3)$, cultivated on the production medium for 21 days, were collected by centrifugation $\left(10 \mathrm{~min}, 4000 \times g, 4^{\circ} \mathrm{C}\right)$. The pellets were dissolved in a lysis buffer (20 mM Tris; $\mathrm{pH} 7 ; 0.5 \mathrm{mM} \mathrm{MgCl} 2 ; 5 \%$ glycerol; and 1 tablet of cOmplete protease inhibitor cocktail (Roche) per $50 \mathrm{~mL}$ buffer) and added into a $2 \mathrm{~mL}$ tube of lysing matrix C (MP biomedicals) containing $1 \mathrm{~mm}$ silica spheres and subjected to 2 cycles of $6 \mathrm{~m} \cdot \mathrm{s}^{-1}$ in a FastPrep Celldisrupter (MP biomedicals). The crude lysates were subjected to centrifugation $\left(10 \mathrm{~min}, 4000 \times g, 4^{\circ} \mathrm{C}\right)$. The resulting supernatants (lysate preparations) were transferred into $2 \mathrm{~mL}$ Eppendorf tubes ( $1 \mathrm{~mL}$ at $1 \mathrm{mg} \cdot \mathrm{mL}^{-1}$ protein), supplemented with $2 \mathrm{mM}$ of SLs and incubated in triplicate $(n=3)$ at $30{ }^{\circ} \mathrm{C}$ on a rotary wheel at $30 \mathrm{rpm}$ for 6 days for di-acetylated lactonic and 9 days for non-acetylated acidic SLs, respectively. For each assay, two controls were included as already described for the 
secretomes, summarized as follows: $2 \mathrm{mM}$ of SLs in lysis buffer and $\mathrm{dH}_{2} \mathrm{O}$ (instead of SLs) added to the lysate preparation.

\subsection{Product Identification and Quantification}

Analytical samples (except the samples of the concentrated assays, see below) were prepared by mixing 2 volumes of ethanol with 1 volume of the culture broth after which the mixture was shaken vigorously for $5 \mathrm{~min}$. The mixture was centrifuged $(10,000 \mathrm{rpm}$ for $5 \mathrm{~min}$ ), and the clear supernatant consisting of a $\mathrm{EtOH} / \mathrm{H}_{2} \mathrm{O} / \mathrm{SL}$ mixture was analyzed after filtration with a $13 \mathrm{~mm}$ PTFE syringe filter with a pore size of $0.2 \mu \mathrm{m}$ (Novolab). For the samples of the assays (with concentrated secretomes and cell lysates), hydroxylated lauric acid (50 nmol) was added as an internal standard before extraction: $440 \mu \mathrm{L}$ ethyl acetate and $11 \mu \mathrm{L}$ acetic acid were added to $1 \mathrm{~mL}$ of the assay volume and shaken vigorously for $5 \mathrm{~min}$. After centrifugation (10,000 rpm for $5 \mathrm{~min}$ ), the upper solvent layer was removed and translocated into a fresh Eppendorf tube, containing $700 \mu \mathrm{L}$ of ethanol and filtered before analysis.

HPLC-ELSD (Varian Prostar HPLC system) analyses were performed as described by Saerens et al. [51]. The same column and LC conditions were used for performing HPLC-MS analyses, using an Intertek ASG (Manchester, UK) with a Micromass Quattro Ultima LIMS1107 (Waters). The detection range was set at $m / z 100$ to 1000, and the negative ion mode was applied.

A second HPLC-MS method was used for sophorose detection. The used column was a Hypercarb PGC $100 \times 4.6 \mathrm{~mm}$ column from Thermo. Three eluents were used: $4 \%$ methanol in ultrapure water (A), $100 \%$ acetonitrile (B), and $15 \%$ formic acid in ultrapure water (C) on a flow rate of $0.6 \mathrm{~mL} \cdot \mathrm{min}^{-1}$ and run time of $40 \mathrm{~min}$. The analysis started at $100 \%$ of eluent $\mathrm{A}$ for $4 \mathrm{~min}$, after which a linear decrease of $\mathrm{A}$ to $78 \%$ and respective increase of $B$ to $22 \%$ were executed in $5 \mathrm{~min}$. This was followed by a further decrease of $A$ to $41 \%$ and respective increase of $C$ to $37 \%$ in the next $8 \mathrm{~min}$ after which eluent $A$ decreased further to $3 \%$ in the next $10 \mathrm{~min}$, whereas $\mathrm{C}$ increased to $75 \%$ and $\mathrm{B}$ was kept at $22 \%$. After this, $A$ and $B$ were increased again to $50 \%$ in $2 \mathrm{~min}$, and C dropped back to $0 \%$. The next minute served to raise A back to $100 \%$, which was kept that way for the last $10 \mathrm{~min}$ of the run. A sophorose standard of $250 \mathrm{mM}$ was used.

UPLC-ELSD (Waters Acquity H-class system) analyses were performed as described by Van Renterghem et al. [16]. For UPLC-HRMS (Thermo ScientificTM ExactiveTM Plus Orbitrap Mass Spectrometer), the same LC method was used, and high-resolution scanning happened in the negative ionization mode (ESI) for a mass range of 215 to $1800 \mathrm{~m} / z$.

For a quick follow up of SL production and substrate use, TLC was performed on the culture broth samples ( 2 to $4 \mu \mathrm{L}$ ) as described by Van Renterghem et al. [16].

\section{Results}

As already mentioned in the introduction, five hypotheses on the natural role of sophorolipids (SLs) for S. bombicola have been postulated. Four of those statements were investigated in this paper: (1) the antimicrobial activity of SLs, (2) the protection against high osmotic pressure, (3) an improved uptake of hydrophobic substrates, and (4) an extracellular storage compound.

\subsection{Antimicrobial Activity}

SLs are claimed to possess antimicrobial activity and as such favor the producing organism against competing microorganisms occupying the same ecological niche. Antimicrobial activity was first reported for lactonic SLs that inhibited growth of specific alkane utilizing yeasts (only when grown on C10 to C18 alkanes) [31]. Succeeding studies showed that SLs also inhibit bacteria (e.g., B. subtilis and P. aeruginosa) and fungi (e.g., U. maydis or foodborne Aspergillus strains) [15,32-37]. Here, the antimicrobial properties of SLs were investigated against some standard microbial strains used for antimicrobial studies and representing different microbial groups, and three ecologically highly relevant strains. 
An in vitro antimicrobial assay was thus performed to determine the minimum inhibitory concentrations (MIC) of a S. bombicola wild-type SL mixture (acidic and lactonic SLs) and two purified components: di-acetylated lactonic SLs and non-acetylated acidic SLs. Especially purified lactonic SLs are interesting to test, as they are the main products that are found extracellularly, where they should exert their antimicrobial activity as a natural function. The antimicrobial activity was tested against species belonging to genera that are associated with flowers and (bumble)bees: Gram+ bacteria (B. subtilis, S. aureus, F. fructosus), Gram- bacteria (E. coli, P. aeruginosa, H. alvei), and yeasts (C. albicans, Z. rouxii) (see Figure 2) [52-55]. Three strains in particular are natural competitors in the ecological niche of S. bombicola as they were all isolated from the gut of bumblebees (and from honey and/or floral nectar): Fructobacillus fructosus, Hafnia alvei, and Zygosaccharomyces rouxii [56-58]. Due to solubilization difficulties in water, SLs were dissolved in DMSO. To check whether the inhibitory effect could be attributed to the SLs and not to DMSO, a preliminary test was performed for each strain to determine the maximal DMSO concentration that did not affect growth. These concentrations of DMSO were included in the assays as controls. Since the pure DMSO concentrations corresponding to the maximum tested SL concentrations did not inhibit growth (data not shown), the observed inhibitory effects can be attributed to the SLs.

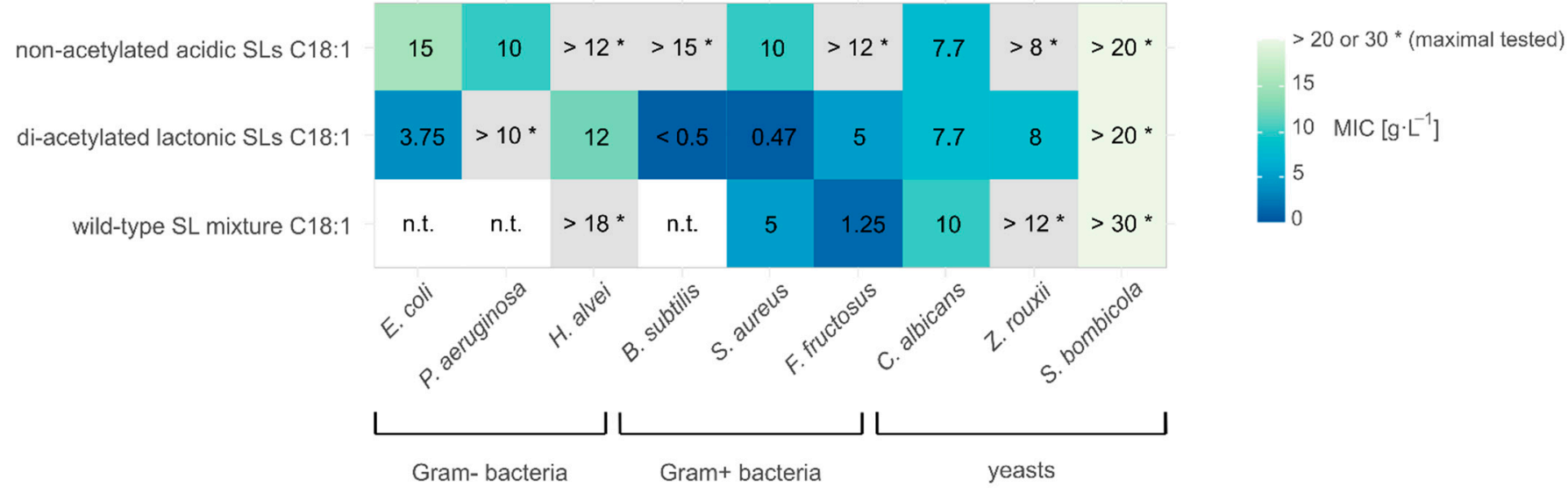

Figure 2. A heatmap of the minimum inhibitory concentrations (MIC) in $\mathrm{g} \cdot \mathrm{L}^{-1}$ found for the wild-type sophorolipid mixture (C18:1) and two of its (main) components: di-acetylated lactonic and non-acetylated acidic SLs. For the combinations marked with a * no MIC value could be determined within the tested concentration range (maximum tested value is depicted on the heatmap), and combinations that were not tested are designated as n.t. MIC values were determined in duplicate $(n=2)$ and were equal for each replicate.

In general, the lactonic SLs exerted the highest antimicrobial activity, while acidic SLs or the wild-type SL mixture showed the strongest inhibition for P. aeruginosa and F. fructosus, respectively. MLC values (minimum lethal concentration) could only be determined for $S$. aureus, F. fructosus, and Z. rouxii within the tested SL concentration range, with a MLC of 15 and $8 \mathrm{~g} \cdot \mathrm{L}^{-1}$ di-acetylated lactonic SLs for S. aureus and Z. rouxii, respectively, and a MLC value of the $10 \mathrm{~g} \cdot \mathrm{L}^{-1}$ wild-type SL mixture for F. fructosus. For all tested microorganisms, at least one MIC value could be determined within the tested range of one of the compounds. S. bombicola on the other hand was not inhibited by the addition of SLs within the tested range, granting it a competitive advantage, which thus indeed confirms this to be a plausible natural function or advantage of SLs for S. bombicola.

\subsection{Protection against Osmotic Pressure}

Hommel et al. (1994) suggested that SLs produced by S. apicola aid in the adaptation of the yeast to high osmotic pressure, arising from the prevalence of sugars in honey and nectar, characterizing its natural environment (see Table 1). They noted parallels in the biosynthesis of the sophorose moiety of SLs and trehalose synthesis in S. cerevisiae [38]. 
The latter is known to act as a compatible solute under (osmotic) stress conditions [59]. However, compatible solutes are defined as highly water soluble and low molecular mass molecules that accumulate to high intracellular concentrations, three conditions that are not fulfilled for SLs [60]. Inverting this rationale, one could theoretically imply a relief of osmotic stress: the osmotically active, highly soluble, low molecular weight sugars outside the cell are converted into high molecular weight and less soluble SLs, thereby alleviating the osmotic pressure on the microbial cell. If this process represents (one of) the natural function(s) of SLs, it should entail enhanced growth of a strain capable of making SLs compared to a strain deficient in SL biosynthesis when grown on high sugar concentrations, which will be investigated here.

To evaluate if sophorolipids (SLs) aid S. bombicola to withstand osmotic pressure caused by the prevalence of sugars in its natural environment (mainly fructose, glucose, and sucrose), a growth experiment was carried out with the SL deficient $\Delta c y p 52 M 1$ S. bombicola strain and the wild-type S. bombicola strain on increasing concentrations (up to $600 \mathrm{~g} \cdot \mathrm{L}^{-1}$ ) of glucose or fructose. The results of the determined growth parameters (maximum growth rate $\mu_{\max }\left(\mathrm{h}^{-1}\right)$, the duration of the lag phase $(\mathrm{h})$, and the $\left.\Delta \mathrm{OD}\right)$ are depicted in Figure 3. There were no significant differences observed in the growth profiles and parameters between the SL deficient S. bombicola $\triangle$ cyp52M1 strain and the wild-type S. bombicola strain, except for the $\triangle \mathrm{OD}$ values obtained on $20 \mathrm{~g} \cdot \mathrm{L}^{-1}$ fructose and glucose, and $120 \mathrm{~g} \cdot \mathrm{L}^{-1}$ fructose, with p-values of $0.04,0.02$, and 0.02 , respectively. For the latter cases, the wild type was able to reach slightly higher OD values/cell densities, suggesting a small fitness cost of the knockout of the cyp52M1 gene. An analysis of the end cultures $(t=60 \mathrm{~h})$ grown on $120 \mathrm{~g} \cdot \mathrm{L}^{-1}$ glucose or fructose confirmed de novo sophorolipid production for the wildtype strain in contrast to a lack of SL production in the S. bombicola $\Delta c y p 52 M 1$ strain (see Figure S1). However, this production of SLs did not result in better growth in comparison to the condition where they were not produced, as growth declined for both strains when sugar concentrations increased with almost no growth observed on $600 \mathrm{~g} \cdot \mathrm{L}^{-1}$ of sugar. Interestingly, fructose seems to give rise to better growth than glucose, especially at higher concentrations, which corresponds with the fact that $S$. bombicola is a fructophilic yeast (preference for fructose over glucose) [61]. Microscopic observations of the end cultures also showed no aberrant morphological differences between a wild-type and a $\triangle c y p 52 M 1$ strain, nor between low or high sugar concentrations (see Figure S2). At 400 and $600 \mathrm{~g} \cdot \mathrm{L}^{-1}$ of glucose or fructose, distinctly less to no cells were visible, corresponding to the lower OD values which were therefore not caused by cell shrinkage. Similar growth profiles were noticed when sucrose was used as the carbon source when molecular masses are considered (e.g., the growth curve on $800 \mathrm{~g} \cdot \mathrm{L}^{-1}$ of sucrose coincides with the one on $400 \mathrm{~g} \cdot \mathrm{L}^{-1}$ of glucose; see Figure S3). This could be expected as osmotic pressure is a colligative property, which means it only depends on the amount of dissolved solute molecules. These results indicate that the capability of producing SLs does not entail protection against high osmotic pressure, at least not under lab conditions.

\subsection{Uptake of Hydrophobic Substrates}

In early research, the production and secretion of SLs (and biosurfactants in general) were described in relation to the growth on hydrophobic carbon sources and were considered as a means for the producing organisms to effectuate the catabolization of hydrophobic substrates such as alkanes, whether or not through their emulsifying properties [39,40]. However, Hommel et al. later stated that the biosynthesis of SLs by Starmerella yeasts cannot simply be a prerequisite for the degradation of extracellular hydrocarbons, as SL production based on glucose (and other sugars) alone was reported for S. apicola and S. bombicola, independent of the fact that hydrocarbons were present or not $[38,62]$. Moreover, the evaluated alkanes in the literature in this respect are not typically present in the natural habitat of S. bombicola where SL biosynthesis has evolved. Therefore, two types of natural accessible hydrophobic substrates present in the habitat of S. bombicola were 
evaluated in this study: beeswax and triacylglycerols of rapeseed oil (plant oil), alongside hexadecane as a control from the literature.

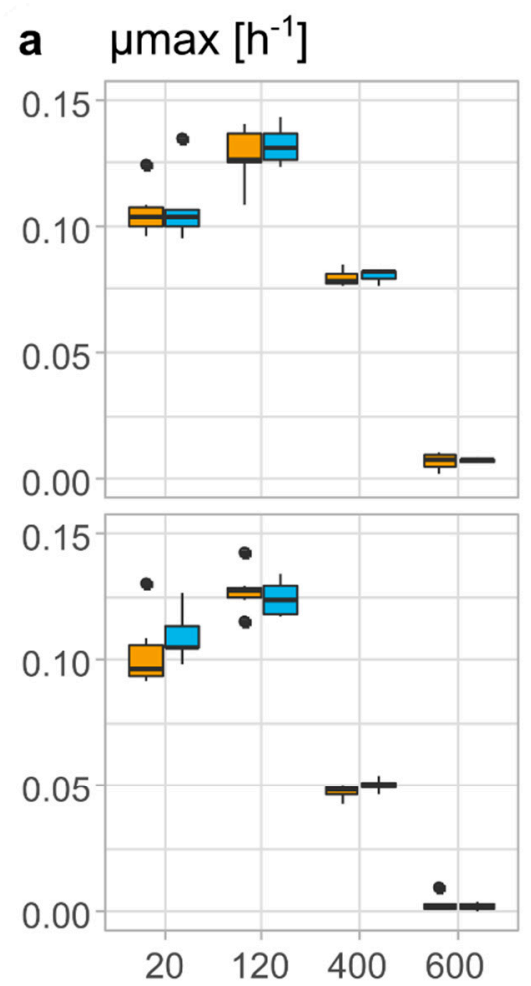

b $\operatorname{lag}[\mathrm{h}]$
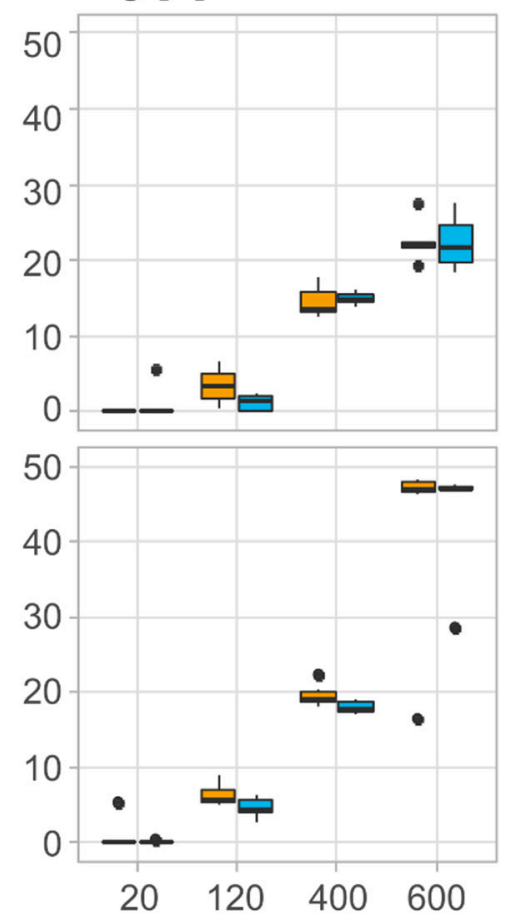

concentration $\left[\mathrm{g} \cdot \mathrm{L}^{-1}\right]$ c $\triangle \mathrm{OD}$
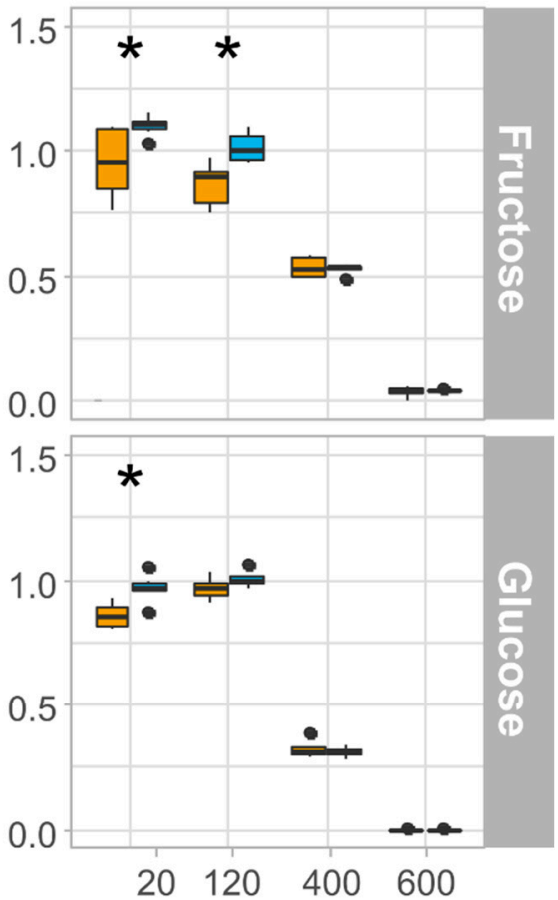

S. bombicola $\triangle$ cyp52M1

S. bombicola wild type

Figure 3. Boxplots of the growth parameters of a sophorolipid deficient S. bombicola $\Delta c y p 52 M 1$ strain (yellow boxes, left) and a sophorolipid producing S. bombicola wild-type strain (blue boxes, right) on increasing concentrations ( $20 \mathrm{~g} \cdot \mathrm{L}^{-1}$ to $\left.600 \mathrm{~g} \cdot \mathrm{L}^{-1}\right)$ of fructose (top) and glucose (bottom) $(n=6)$. (a) The maximal growth rate $\mu_{\max }\left(\mathrm{h}^{-1}\right),(\mathrm{b})$ the duration of the lag phase $\left(\mathrm{h}\right.$ ), and (c) the $\triangle \mathrm{OD}$ reached during growth (measured $\mathrm{OD}_{600}$ value of the stationary phase minus value at $\mathrm{t}_{0}$ ). Significant differences in growth between both strains are marked on top of the corresponding boxes: ${ }^{*} 0.01<p \leq 0.05$.

To examine if the presence of SLs enhances the catabolization of these hydrophobic substrates, most likely through solubilization/emulsification effects exerted by SLs, a growth experiment was performed with $20 \mathrm{~g} \cdot \mathrm{L}^{-1}$ of beeswax, rapeseed oil, or hexadecane as the sole carbon source. Both a SL producing wild-type and a SL deficient $\Delta c y p 52 M 1$ strain [47] were evaluated in the absence and presence of $1 \mathrm{~g} \cdot \mathrm{L}^{-1}$ of the wild-type SLs mixture, and several growth parameters were estimated: the estimated growth rate $\mu\left(\mathrm{h}^{-1}\right)$, the duration of the lag phase (d), and the $\triangle \mathrm{OD}$ (corresponding to the produced biomass). The cells were cultured in glass tubes because of the inability to grow on rapeseed oil in deep well plates (a substrate that certainly can be metabolized by a wild-type S. bombicola), probably due to altered oxygen availability. Consequently, it was hard to keep the cultures sterile for longer than 7 days when growth was lagging. Therefore, growth parameters were calculated based on OD measurements up to day 7 for beeswax and hexadecane, and up to day 14 for the inoculated cultures on rapeseed oil. No growth was observed for the inoculated negative controls (on the medium without a $\mathrm{C}$-source, with and without the addition of SLs) for at least 7 days.

For beeswax - a solid substrate consisting of a complex mixture of long chain monoesters (C40-C48), hydrocarbons (C27-C33), and fatty acids (C24-C32) - no growth was visible after 7 days for either of the two $S$. bombicola strains, neither in the presence or 
absence of SLs (Figure 4). S. bombicola is not able to use beeswax as a sole carbon source (at least not under submerged lab conditions after 7 days of incubation).

a

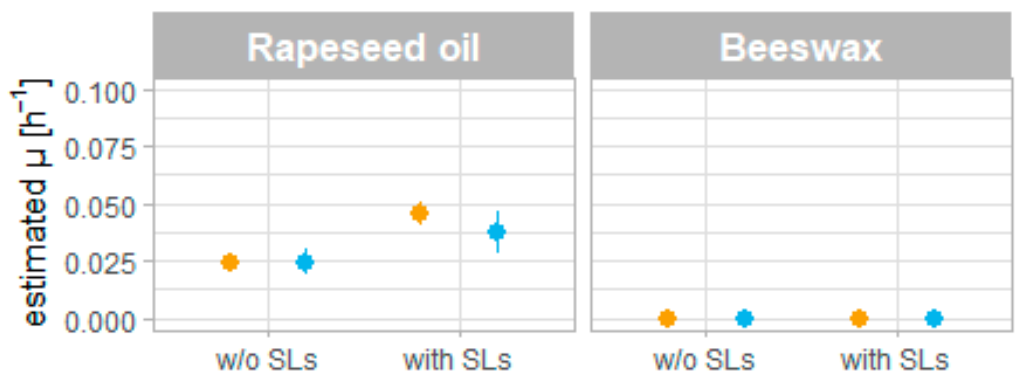

b

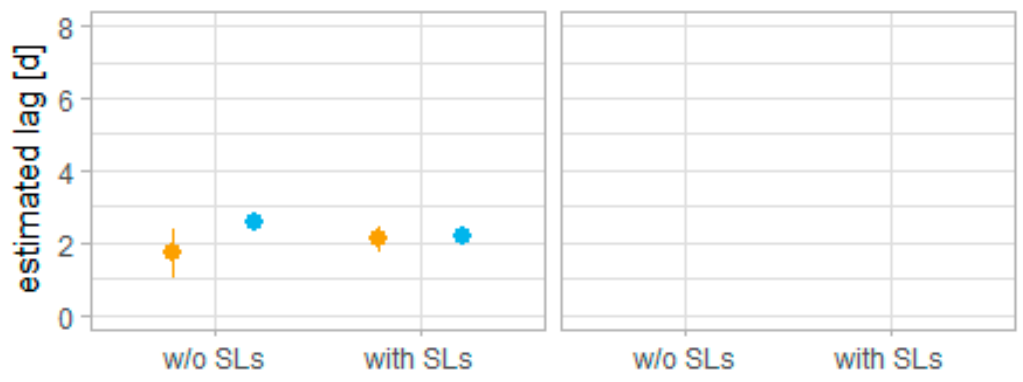

C

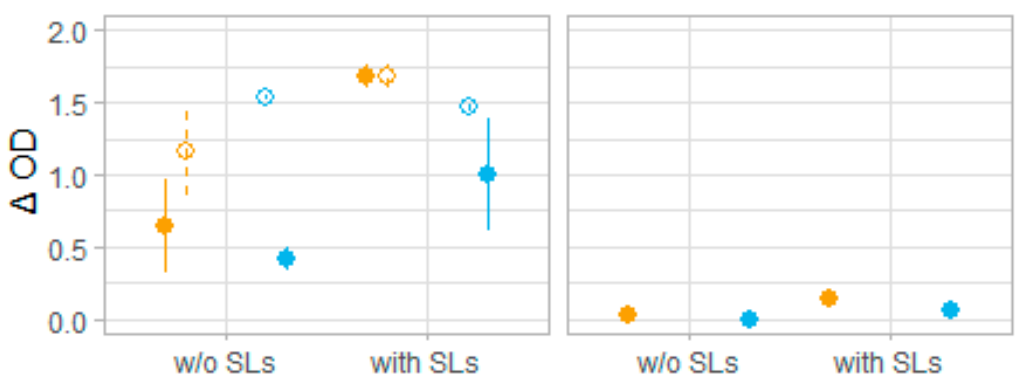

\section{S. bombicola $\triangle$ cyp52M1 S. bombicola wild type}

Figure 4. Scatterplots of the estimated growth parameters of a sophorolipid deficient S. bombicola $\triangle c y p 52 M 1$ strain (yellow dots, left) and a sophorolipid producing S. bombicola wild-type strain (blue dots, right) on rapeseed oil $(n=3)$ and beeswax $(n=2)$ in the presence ('with') or absence (' $\left.w / o^{\prime}\right)$ of $1 \mathrm{~g} \cdot \mathrm{L}^{-1}$ wild-type sophorolipid mixture. The depicted error bars represent the standard deviation. (a) The estimated growth rate $\mu\left(\mathrm{h}^{-1}\right)$, (b) the estimated duration of the lag phase (d) (cultures that lagged until the end of the experiment are not depicted on the plot), and (c) the $\triangle \mathrm{OD}$ (maximum measured $\mathrm{OD}_{600}$ value minus value at $\mathrm{t}_{0}$ ) at day 7 (full dots) and for rapeseed oil also at day 14 (empty dots).

On the other hand, both the wild-type and the $\Delta$ cyp52M1 S. bombicola strains were able to grow on rapeseed oil—consisting of triacylglycerols of mainly oleic, linoleic, and linolenic acid-implying that SLs are no prerequisite for the catabolization of rapeseed oil. The addition of $1 \mathrm{~g} \cdot \mathrm{L}^{-1}$ of SLs did not decrease the duration of the lag phase (Figure $4 \mathrm{~b}$ ), but slightly increased the growth rate (Figure $4 a$ ), although this difference is only significant for the $\Delta$ cyp52M1 strain: $p=0.03$ vs. $p=0.14$ for the wild type. One could argue that this observation could be caused by the fact that SLs are also produced by the wild type (SL 
production was confirmed at day 14; see Figure S4) and hence already promote growth without the additional supplemented SLs. Nevertheless, the estimated $\mu$ of the wild type was not higher than the $\mu$ of the $\Delta c y p 52 M 1$ strain, and the presence of SLs in the culture broth could not be confirmed yet after 7 days (TLC, see Figure S4). Improving the statistical power by increasing the sample size might help if there was an undetected significant difference for the wild type (with and without the addition of SLs) in this experiment. The higher growth rates for the cultures with added SLs are also reflected in the $\triangle \mathrm{OD}$ values (equaling the total grown biomass) at day 7, with a $p$ value of 0.03 for the $\Delta c y p 52 M 1$ strain and 0.09 for the wild type (see Figure 4c). The cultures with added SLs already reached the stationary phase after 7 to 9 days, while some of the cultures without SLs did not even reach the stationary phase after 14 days (see Figure S5).

These observations support the hypothesis that SLs can promote growth on some hydrophobic substrates, in this case rapeseed oil, probably through a solubilization effect. This theory however cannot be generalized to all hydrophobic substrates: no growth was observed after 7 days on hexadecane as the sole C-source when SLs were added for both the wild-type and the $\Delta c y p 52 M 1$ S. bombicola strains, although without SLs the estimated growth rates $\mu$ were higher than for rapeseed oil (see Figure S6). Additionally, the ability of producing SLs is clearly not sufficient to promote growth on rapeseed oil directly, as the growth of the wild type is not surpassing that of the SL deficient $\Delta c y p 52 M 1$ strain (in both $\mu$, lag, and $\Delta \mathrm{OD}$ ) in the first 7 days; only when SLs were already present/added in the pre-exponential phase a beneficial effect was noticed, indicating that growth on hydrophobic substrates is a less important physiological function of SLs.

\subsection{Exclusive Storage Compound}

The possibility that one of the functions of SLs could be the formation of an extracellular storage compound has long been underexposed. However, converting an easily accessible carbon source such as glucose or fructose (present in honey and nectar) into an energy-rich yet metabolically less accessible molecule such as sophorolipids is an excellent mechanism to compete with other organisms populating the same habitat. In this way, the organism can monopolize the carbon source present in the environment and simultaneously build up an energy reserve that can be utilized later on. This function of SLs was first theoretically postulated by Hommel et al. (1994), as trehalose not only functions as a compatible solute in S. cerevisiae (see above) but also acts as a storage compound $[38,63]$. This theory thus implies that $S$. bombicola should be able to catabolize its own SLs. This was indeed suggested by Garcia-Ochoa et al. (1996) who claimed that the SL concentration decreased from 5 to $1 \mathrm{~g} \cdot \mathrm{L}^{-1}$ when used as the sole carbon source, although later on other authors claimed that S. bombicola is not able to metabolize SLs [64-66].

The idea that SLs—categorized as secondary metabolites—could serve as an extracellular storage compound is emboldened by similar indications and/or proof for other biosurfactants/glycolipids: cellobiose lipids [67], surfactin [68], and mannosylerythritol lipids (MELs) [69]. Moreover, this theory also holds for Pseudohyphozyma bogoriensis as the disappearance of its branched SLs from an old culture medium was already reported in 1961 [70]. The possible catabolization of SLs by the producing organism S. bombicola will be investigated and discussed in detail in this research article.

\subsubsection{Growth on Production Medium}

The theory that sophorolipids (SLs) constitute a build-up of carbon and energy in S. bombicola denotes that this yeast should be able to catabolize its own produced SLs. To investigate this, a prolonged combined production and catabolization experiment was performed: S. bombicola was grown on the SL production medium described by Lang (Table 2) (rapeseed oil was added after $48 \mathrm{~h}$ ), and the cultures $(n=3)$ were incubated beyond glucose exhaustion (for 100 days) to follow up typical parameters and to evaluate potential evidence for SL catabolization. The results are shown in Figures 5-7. 


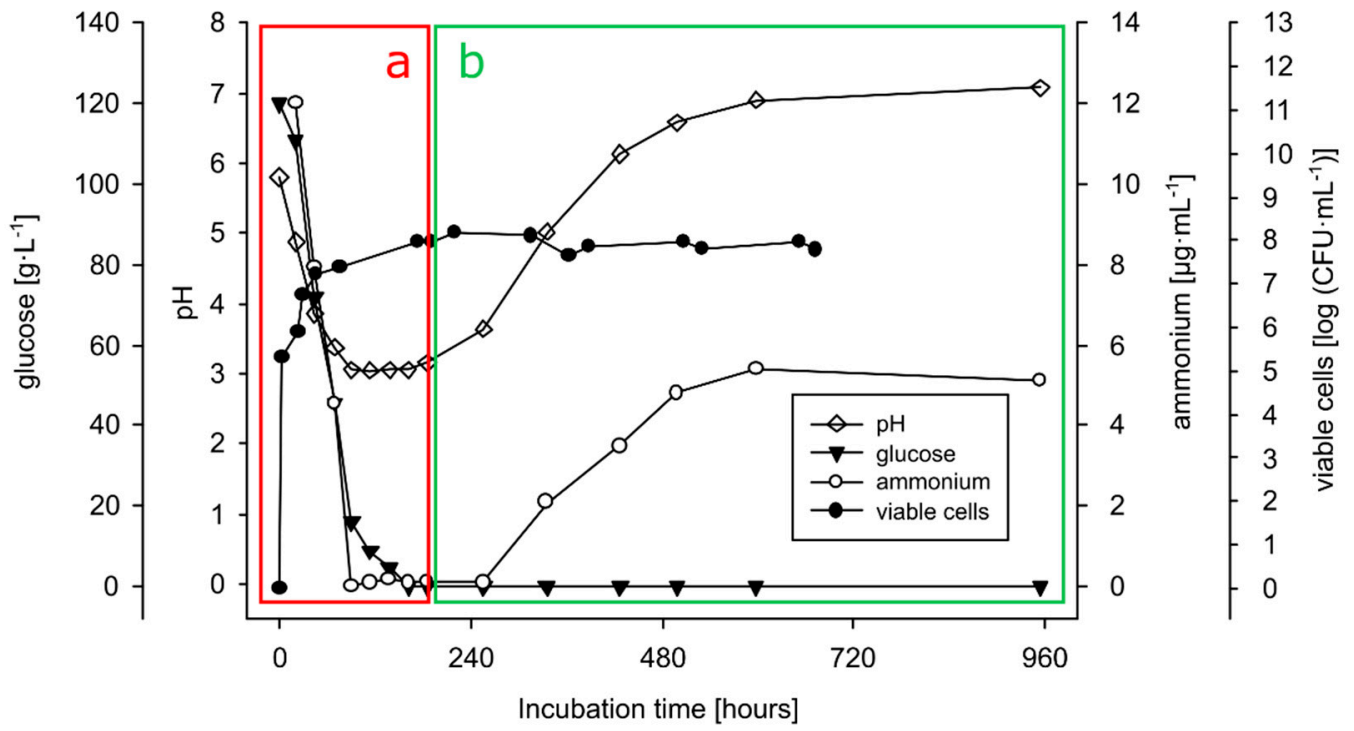

Figure 5. (a) SL biosynthesis and (b) SL catabolization phase of Starmerella bombicola cultivated on the production medium. $(\diamond) \mathrm{pH},(\mathbf{\nabla})$ glucose concentrations, $(\bigcirc)$ ammonium concentrations, and $(\bullet)$ viable cell concentrations are shown.

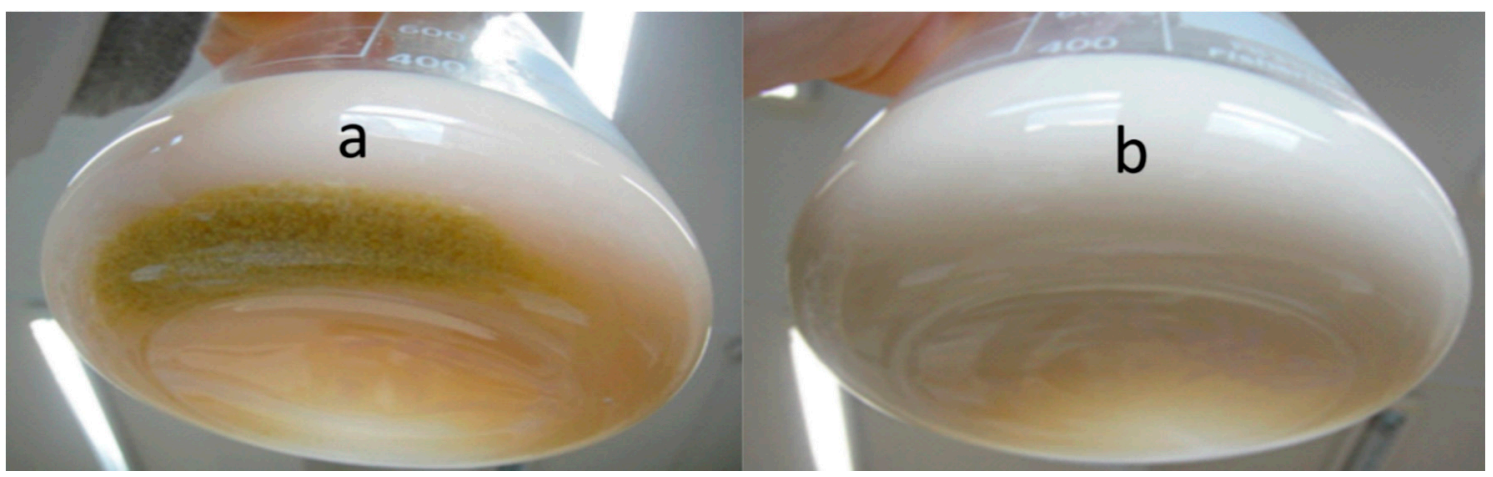

Figure 6. Shake flask culture of Starmerella bombicola cultivated on the production medium after (a) $240 \mathrm{~h}$ (10 days) of cultivation and (b) $960 \mathrm{~h}$ (40 days) of cultivation.

The first phase of the experiment (Figure 5a) can be distinguished as the SL production phase: di-acetylated lactonic SLs (C18:1 and C18:0) were the predominantly produced forms of SLs as shown in Figure 7a. Lactonic SLs are typically the dominant form of SLs produced by S. bombicola on the production medium and start to precipitate after $130 \mathrm{~h}$ ( \pm 5.5 days) (see Figure $6 \mathrm{a}$ ) because their solubility dramatically decreases at lower $\mathrm{pH}$ values (see Table S1). The observed strong $\mathrm{pH}$ decrease (see Figure 5a) resulted from the secretion of organic acids such as citrate and isocitrate (up to $10 \mathrm{~g} \cdot \mathrm{L}^{-1}$ ) and the utilization of the nitrogen source during cell growth [71].

The second phase of the experiment (see Figure $5 b$ ) is initiated upon glucose depletion at about 10 days of cultivation. A steep $\mathrm{pH}$ and ammonium rise were observed. The $\mathrm{pH}$ rise can (partly) be explained by the increase of free ammonium ions, which is the result of the deamination of amino acids once glucose is depleted and the yeast shifts from carbohydrates to proteins as the energy source. The produced organic acids can also be used as a carbon source as the concentrations of citrate and isocitrate were reported to decrease when the glucose in the culture medium was nearly consumed [72]. Remarkably, the precipitated SLs (di-acetylated lactonic form) started to disappear again after $330 \mathrm{~h}$ ( \pm 14 days) of incubation to vanish eventually completely (see Figure $6 b$ ). 


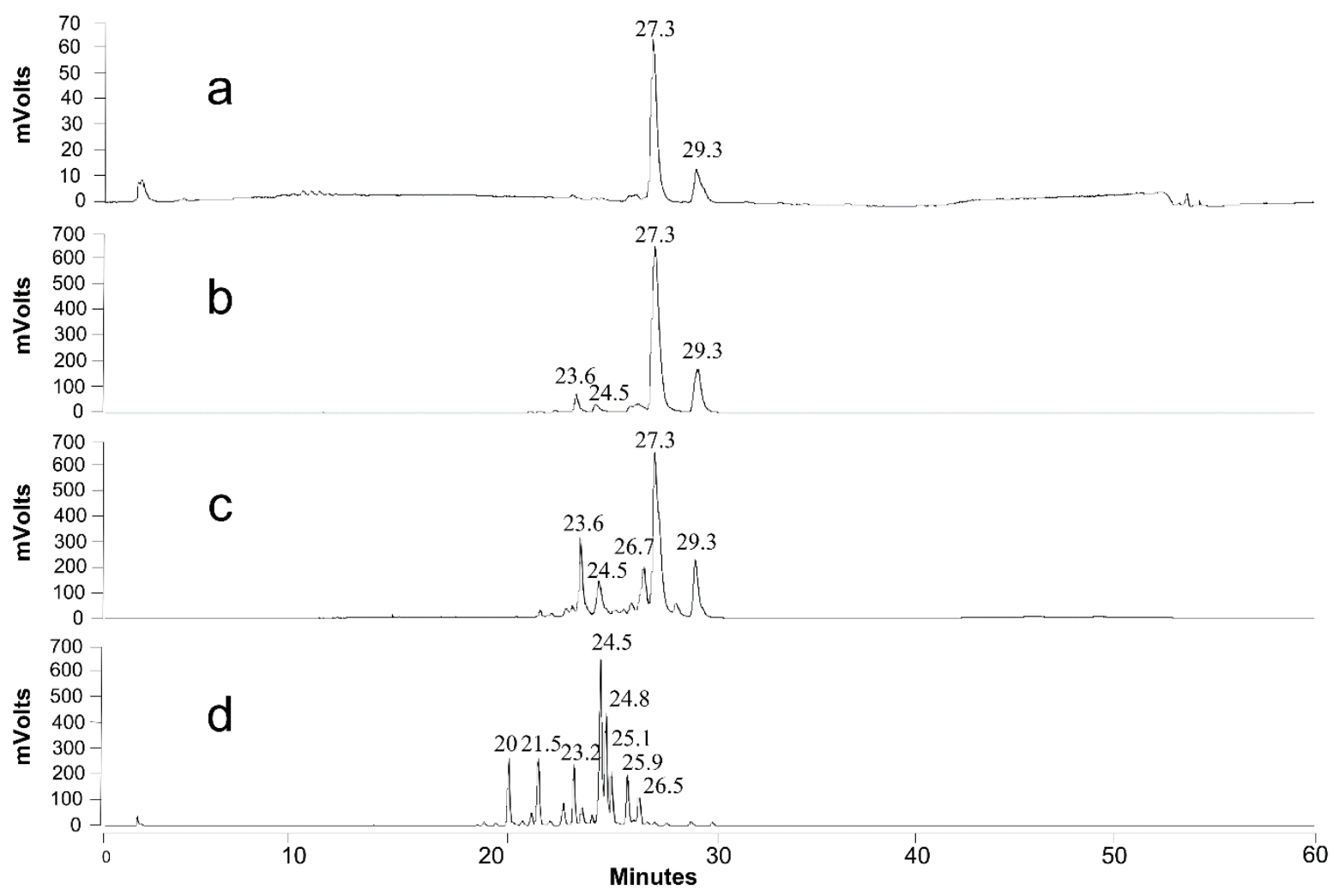

Figure 7. HPLC-ELSD chromatograms from a S. bombicola culture on production medium taken after (a) 8 days, (b) 15 days, (c) 40 days, (d) and 100 days of incubation. Peaks correspond to di-acetylated lactonic SLs C18:0 (29 min) and C18:1 (27 min), mono-acetylated lactonic SLs (23 and $24 \mathrm{~min}$ ), di-acetylated acidic SLs (23 and $24 \mathrm{~min}$ ), mono-acetylated acidic SLs C18:1 (21 min), non-acetylated acids SLs C18:1 (20 min), and hydroxylated fatty acids (25 and $26 \mathrm{~min})$.

Although a rising $\mathrm{pH}$ promotes the solubilization of the precipitated SLs, a LC-MS analysis also showed that the SL mixture became enriched in more hydrophilic compounds: after 15 days, mono-acetylated lactonic SLs (24 min) and di-acetylated acidic SLs (23 min) appeared (Figure $7 \mathrm{~b}$ ), and their concentration increased with time (Figure 7c: 40 days). After 100 days of cultivation (Figure 7d), di-acetylated lactonic SLs nearly completely disappeared (peak at 27 and 29 min for C18:1 and C18:0, respectively) and a complex mixture of compounds was present in the extracellular medium: mono- and di-acetylated lactonic SLs (still present in minor amounts); di-, mono-, and non-acetylated acidic SLs; (acetylated) glucolipids and hydroxylated fatty acids. Remarkably, the log CFU values, a marker for cell viability, remained constant (as shown in Figure 5) for over 100 days without the substrate addition ( $\log \mathrm{CFU}$ values at $960 \mathrm{~h}$ could not be determined due to contamination on the dilution plates; $\log \mathrm{CFU}$ values at day 100 are not shown). These observations thus clearly indicate that $S$. bombicola can catabolize SLs in times of starvation. However, it is obviously plausible that the incubation of solubilized SLs in the watery medium environment at $30^{\circ} \mathrm{C}$ and neutral $\mathrm{pH}$ values for over 3 months could give rise to spontaneous hydrolysis. Especially ester functionalities (acetyl- and lacton functions) are quite prone to spontaneous hydrolysis in water, particularly at $\mathrm{pH}$ values above 6 . This thus required further investigation.

\subsubsection{Growth on Sophorolipids as the Sole Carbon Source}

If the disappearance of sophorolipids (SLs) during prolonged incubation as described above is caused by SL catabolization, S. bombicola should be able to grow on the medium with SLs as the sole carbon source (called the SL medium). A specific cultivation experiment to investigate this was set up as described under the methods and materials section. After three to four days of cultivation, multipolar budding of S. bombicola was indeed observed, 
although high cell densities were not attained. The observed growth could clearly be linked to the consumption of the added SLs by S. bombicola, as after 1 month of cultivation, almost all the SLs and derivatives disappeared from the extracellular culture medium (Figure S7d and Table S2).

Similar to the observations of the first experiment described above, the first two catabolic activities that were detected were deacetylation and the ring-opening of the SLs resulting in mono-acetylated lactonic SLs and di-acetylated acidic SLs, respectively (Figure S7a). Longer cultivation gave rise to a further breakdown of SLs into mono- and non-acetylated acidic SLs, and minor amounts of non-acetylated lactonic SLs (C18:1) were also detected (Figure S7b). After 30 days of cultivation, almost all the SLs disappeared from the culture medium (Figure S7d). Importantly, near to no hydrolysis of SLs was observed in the controls (incubation of the cell free SL medium) (similarly as shown in Figure 8, right). The $\mathrm{pH}$ in the cultures only dropped from 6.06 to 5.60 during cultivation (after 16 days), so spontaneous hydrolysis of the ester functionalities, which can occur at higher and very low $\mathrm{pH}$ values, can also not be argued.

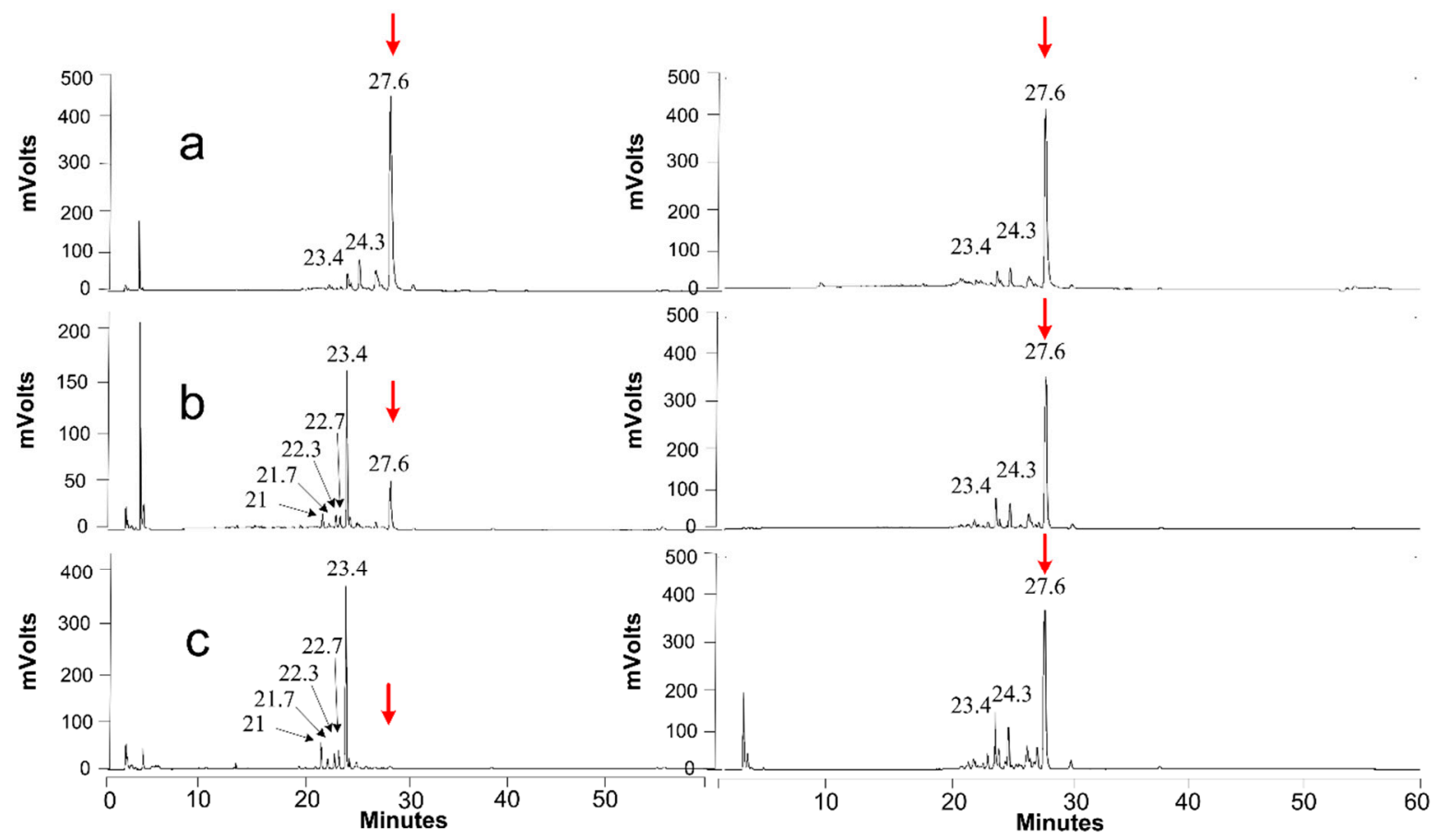

Figure 8. (left) HPLC-ELSD chromatograms of samples from the extracellular activity assay (unconcentrated secretome) incubated with di-acetylated lactonic SLs (27.6 $\mathrm{min}$ ) after (a) $1 \mathrm{~h}$, (b) 10 days, and (c) 32 days of incubation; (right) controls for SLs at each time point. Peaks at $23.4 \mathrm{~min}=$ co-eluting di-acetylated acidic SLs and mono-acetylated lactonic SLs; other peaks at 20-23 $\mathrm{min}=$ mono- and non-acetylated acidic SLs.

These results thus not only affirm the catabolization of SLs by S. bombicola, but also suggest the presence of extracellular enzyme(s) responsible for part of these catabolic processes: one or more hydrolyzing enzymes responsible for the ring-opening of the lactonic SLs and deacetylation of lactonic and acidic SLs. Moreover, in contrast to the first experiment, no glucolipids, hydroxylated fatty acids (nor glucose or sophorose) were detected in the culture medium. This suggests that non-acetylated acidic SLs are taken up by the starved cells to be further metabolized intracellularly.

\subsubsection{Extracellular Activity (of the Secretome)}

To further investigate the catabolic steps, unconcentrated secretomes $(n=3)$ were prepared (from 21-day-old production cultures) and incubated with di-acetylated lactonic or non-acetylated acidic SLs. 
For di-acetylated lactonic SLs, extracellular conversion into di-acetylated acidic and mono-acetylated lactonic SLs was detected after 10 days (both compounds co-eluted at $23.4 \mathrm{~min}$; see Figure $8 \mathrm{~b}$ ). The conversion was complete after 32 days of incubation (see Figure $8 \mathrm{c}$ ). The peaks appearing between 20 and 23 min correspond to mono- and nonacetylated acidic SLs. Some ring-opening and deacetylation was also observed for the control incubation of SLs (see Figure 8, right panels), but these spontaneous effects were clearly a lot less pronounced than those for the incubation with unconcentrated secretomes (the $\mathrm{pH}$ remained stable at about 5.5 for all the assays and controls).

For non-acetylated acidic SLs, no extracellular conversion was observed (see Figure 9). This indicates that these non-acetylated acidic molecules are the end-products of the extracellular catabolization of SLs by S. bombicola. They are thus hypothesized to be taken up again by the starved cells to be further catabolized intracellularly, explaining their disappearance from cultures cultivated on SLs as the sole carbon source (see Figure S7d).

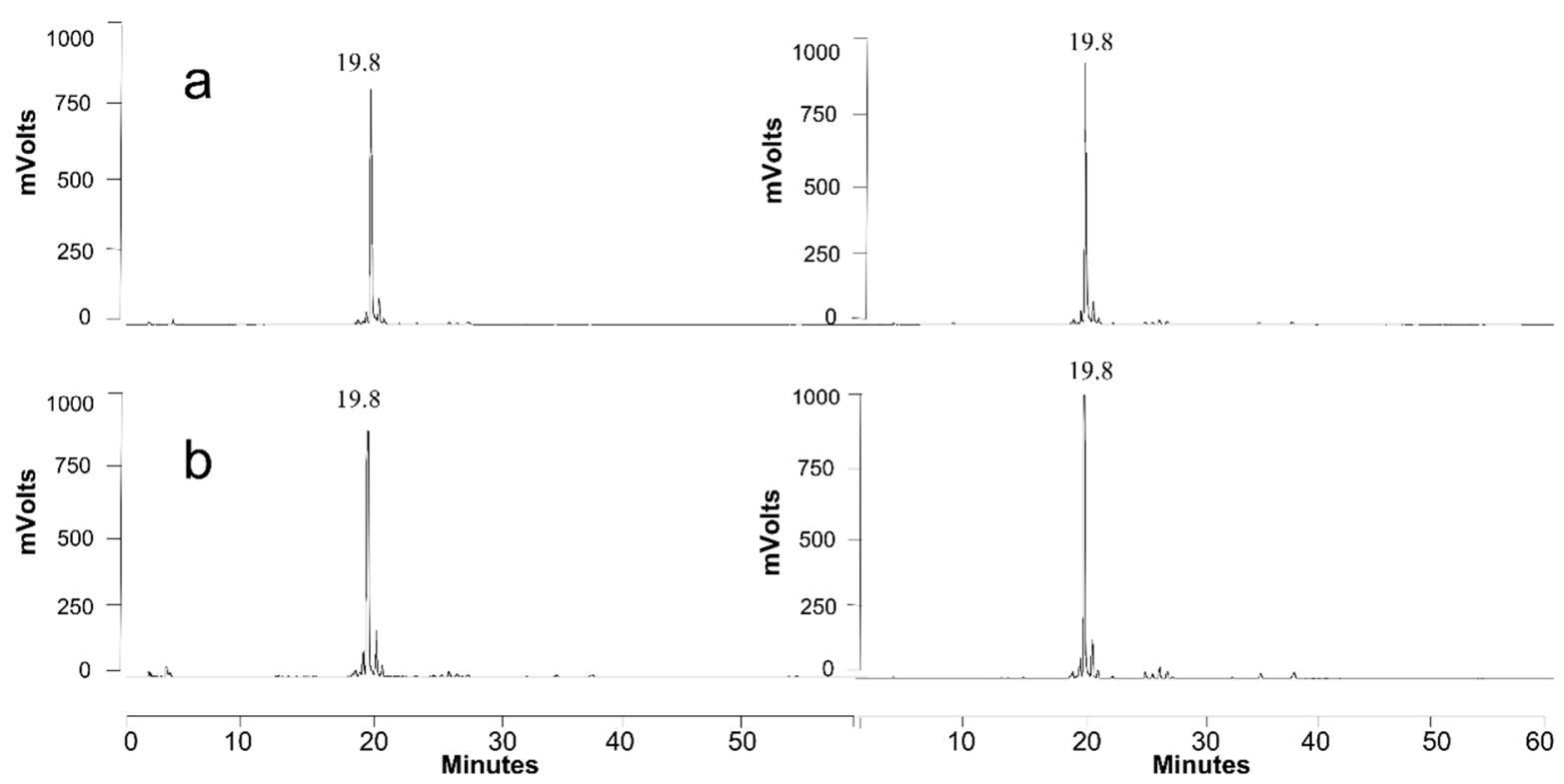

Figure 9. (left) HPLC-ELSD chromatograms of samples from the extracellular activity assay (unconcentrated secretome) incubated with non-acetylated acidic SLs (19.8 $\mathrm{min}$ ) after (a) $1 \mathrm{~h}$ and (b) 16 days of incubation; (right) controls for SLs at each time point.

The obtained results were confirmed by repeating these experiments. In addition, assays with concentrated and standardized protein solutions (secretomes, $\mathrm{pH}$ set on 7) resulted in similar observations. Moreover, when using concentrated secretomes derived from a culture cultivated on SLs as the sole C-source, the demonstrated activity was a lot higher: all of the added substrate (di-acetylated lactonic SLs) was converted into diacetylated acidic SLs and mono-acetylated lactonic SLs after only 6 days of incubation (data not shown). This indicates the upregulation of the responsible gene(s) as the used protein concentrations were constant.

A first 'suspect' for the ring-opening of lactonic SLs that the authors postulated was the S. bombicola lacton esterase enzyme [48]. The sble gene encodes for the enzyme responsible for the lactonization of SLs during the biosynthesis of SLs and could thus be responsible for the opposite reaction as well. To investigate this hypothesis, an additional unconcentrated secretome was prepared of the $\Delta$ sble strain [49] to investigate if ring-opening still occurred. As ring-opening was still observed after 32 days of incubation in a SBLE free secretome, it can be concluded that the SBLE enzyme is not the (only) enzyme responsible for ringopening (see Figure S8). 


\subsubsection{Intracellular Activity (of Cell Lysate)}

To investigate the intracellular degradation of SLs, lysates of wild-type cultures were prepared (from 21-day-old production cultures, $n=3$ ). The assays were incubated for 6 days for di-acetylated lactonic SLs and 9 days for non-acetylated acidic SLs.

Incubation of the lysates with lactonic SLs (Figure S9 and Table S3) gave rise to very similar results as those obtained for the cultivation of S. bombicola on SLs as the sole Csource: ring-opening and deacetylation were observed. All these intermediates already appeared after only $24 \mathrm{~h}$ of incubation, but the effects became more pronounced upon longer incubation. All but one: the emergence of the non-acetylated acidic SLs became only evident after 6 days of incubation. Further disassembly of SLs was not detected, possibly because the incubation time (6 days) was not long enough to allow the build-up of non-acetylated acidic SLs (peak at $19.6 \mathrm{~min}$ ) of which catabolic intermediates could subsequently be derived and detected.

Incubation of the lysate with the non-acetylated acidic SLs $(19.7 \mathrm{~min})$ for 9 days leads to clear degradation: the biggest peaks appearing correspond to C18:1 glucolipids (21.9 $\mathrm{min}$ ) and C18:1 hydroxylated fatty acids $(26.7 \mathrm{~min})$. Other compounds include other hydroxylated fatty acids derived from other SL variants present in the substrate, giving rise to C18:0, C18:2, and C16:0 hydroxylated fatty acids (Figure 10a; and Table 3). These effects were only visible after 6 days of incubation, indicating a slower/less efficient process or lower enzyme concentrations compared to the one described above for di-acetylated lactonic SLs (activity detected after $24 \mathrm{~h}$ at the same total protein concentrations). Together, these results give a strong indication that the catabolization of non-acetylated acidic SLs predominantly occurs intracellularly. None of the abovementioned effects were observed when repeating this experiment with a lysate from a culture that was cultivated for only 4 days (glucose not depleted), so the responsible enzymes are probably not expressed yet.

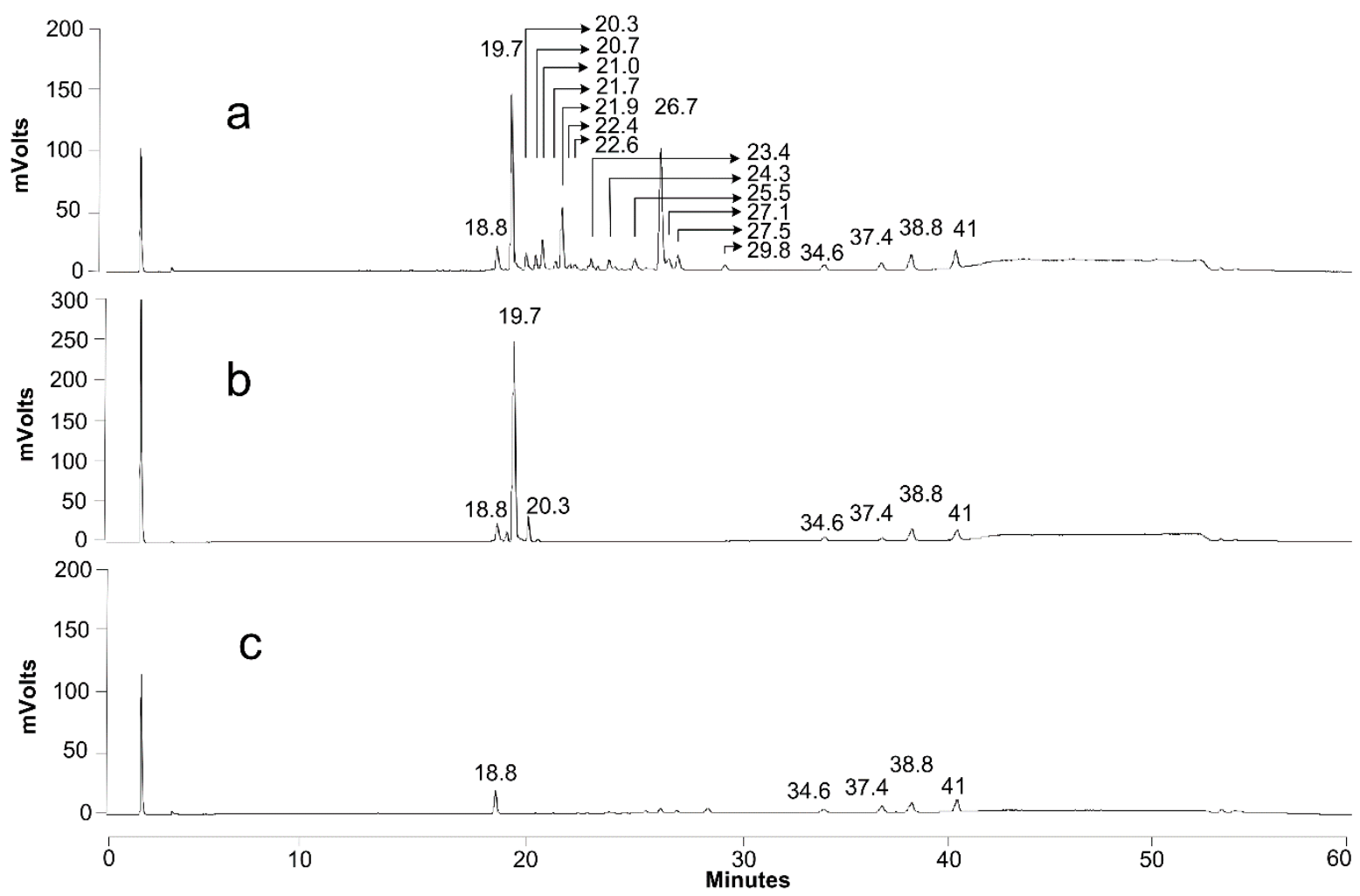

Figure 10. HPLC-ELSD chromatograms of (a) an enzyme assay for intracellular activity (lysate) on non-acetylated acidic SLs (peak at $19.7 \mathrm{~min}$ ) after 9 days of incubation $(\mathbf{b}, \mathbf{c})$ correspond to the controls for SLs and the lysate, respectively. The peak at $18.8 \mathrm{~min}$ corresponds to an internal standard $(\mathrm{C} 12: 0-\mathrm{OH})$. 
Table 3. Identification of the major peaks detected in Figure 10 after HPLC-MS analysis. The detected masses $(\mathrm{m} / \mathrm{z})$ correspond to the $[\mathrm{M}-\mathrm{H}]^{-}$adduct of the identified molecules. The most prominent ones are framed.

\begin{tabular}{ccccc}
\hline Retention Time & $m / z$ & Identity & & Acetylation \\
\hline 18.8 & 215 & hydroxylated fatty acid & $\mathrm{C} 12: 0$ & \\
\hline 19.7 & 621 & acidic SL & $\mathrm{C} 18: 1$ & non \\
\hline 20.3 & 623 & acidic SL & $\mathrm{C} 18: 0$ & non \\
20.7 & 663 & acidic SL & $\mathrm{C} 18: 1$ & mono \\
21 & 459 & glucolipid & $\mathrm{C} 18: 1$ & non \\
21.7 & 665 & acidic SL & $\mathrm{C} 18: 0$ & mono \\
\hline 21.9 & 459 & glucolipid & $\mathrm{C} 18: 1$ & non \\
22.4 & 459 & glucolipid & $\mathrm{C} 18: 1$ & non \\
23.4 & 705 & acidic SL & $\mathrm{C} 18: 1$ & di \\
24.3 & 501 & glucolipid & $\mathrm{C} 18: 1$ & mono \\
25.5 & 295 & hydroxylated fatty acid & $\mathrm{C} 18: 2$ & \\
26 & 271 & hydroxylated fatty acid & $\mathrm{C} 16: 0$ & \\
\hline 26.7 & 297 & hydroxylated fatty acid & $\mathrm{C} 18: 1$ & \\
27.1 & 299 & hydroxylated fatty acid & $\mathrm{C} 18: 0$ & \\
27.5 & 297 & hydroxylated fatty acid & $\mathrm{C} 18: 1$ & \\
29.8 & 299 & hydroxylated fatty acid & $\mathrm{C} 18: 0$ & \\
38.8 & 255 & fatty acid & $\mathrm{C} 16: 0$ & \\
41 & 283 & fatty acid & $\mathrm{C} 18: 0$ & \\
\hline
\end{tabular}

Non-acetylated acidic SLs are thus disassembled intracellularly into glucolipids and hydroxylated fatty acids, suggesting that at least a stepwise detachment of the glucose molecules is occurring. This was confirmed by measuring the glucose concentrations of the end samples (after 9 days of incubation). In contrast, no glucose was detected in the controls, so the glucose released in the assay was clearly derived from hydrolyzed SLs. However, this result does not rule out the possibility that the sophorose molecule can (also) be released, followed by hydrolysis of the disaccharide into two glucose molecules; indeed, minor amounts of a compound corresponding to the mass of sophorose were detected by performing HPLC-MS (data not shown).

One last remarkable observation was that incubation of cell lysates with non-acetylated acidic SLs also resulted in the formation of acetylated intermediates (glucolipids and acidic SLs) (Table 3). These compounds were not present in the controls, and can hence only arise from the action of the acetyltransferase responsible for the acetylation of SLs [73]. This indicates that this enzyme must still be active in the cell lysates of cultures that were cultivated for 21 days. This acetylation was already detected after only $24 \mathrm{~h}$ of incubation, indicating a more efficient biosynthetic process as compared to the catabolic one.

\section{Discussion}

Although potential applications for sophorolipids (SLs) are currently expanding, the physiological role of these SLs for their producing organism Starmerella bombicola remained speculative. Based on their properties, biosynthesis, and some sparse supporting data, five hypotheses could be found scattered around the literature, of which four were investigated in this research article. According to the results presented here, one hypothesis can be discarded: SL production as a protection against osmotic pressure. The other hypothesized natural functions-antimicrobial activity, uptake of hydrophobic substrates, and exclusive storage compound-are supported by our findings.

\subsection{Antimicrobial Activity}

The antimicrobial activity of SLs was already described before. However, varying MIC values are reported for wild-type SL mixtures or one of its components: e.g., for wild-type SL mixtures, MICs are reported of $0.5-10 \mathrm{~g} \cdot \mathrm{L}^{-1}$ for E. coli and of $0.3-4.5 \mathrm{~g} \cdot \mathrm{L}^{-1}$ for S. aureus [74,75]; and for C18:1 non-acetylated acidic SLs and P. aeruginosa, a MLC of 
$5 \mathrm{~g} \cdot \mathrm{L}^{-1}$ was reported, while a MIC of $10 \mathrm{~g} \cdot \mathrm{L}^{-1}$ was found in this study (Figure 2) [15]. These differences can be partly explained by different compositions of the SL mixture (i.e., fatty acid chain lengths, acetylation, and/or lactonization degree and purity), different assay methods, or different tested isolates. However, the determinants defining the antimicrobial activity could be more complex, as Haque et al. found a MIC of $0.06 \mathrm{~g} \cdot \mathrm{L}^{-1}$ for di-acetylated lactonic SLs against C. albicans in comparison to the $7.7 \mathrm{~g} \cdot \mathrm{L}^{-1}$ found in this research paper, using the same isolate and assay but a different medium [36]. Moreover, the MIC value of the wild-type SL mixture for F. fructosus is lower than the MICs of its comprising compounds, suggesting a possible synergistic effect.The natural habitat of $S$. bombicola, i.e., flowers and (bumble)bees, harbors a wide variety of microorganisms [52-57]. Proving that several tested genera, next to three ecologically highly relevant species (H. alvei, F. fructosus, and $Z$. rouxii) are inhibited by SLs correlates with the broad antimicrobial activity that is needed to favor S. bombicola — showing a clear competitive advantage over the other tested microorganisms, as it was not inhibited by 20 or $30 \mathrm{~g} \cdot \mathrm{L}^{-1}$ of SLs-in these environments (see Figure 2). SLs presumably exert their antimicrobial activity by changing or rupturing cellular membranes and hence target a fundamental and universal prerequisite for the survival of cells [76,77]. How S. bombicola acquired an increased resistance and circumvents this antimicrobial mechanism is not clear.

Finally, the fact that SLs are regarded as secondary metabolites also fits with the theory that S. bombicola produces SLs for their antimicrobial activity. Nevertheless, the SL production titers that $S$. bombicola reaches in lab conditions greatly exceed the inhibitory concentrations, which are in turn some orders of magnitude higher than MIC values of 'true antibiotics' (such as penicillin or kanamycin) implying that antimicrobial activity cannot be the sole/main physiological function of SLs [78].

\subsection{Protection against Osmotic Pressure}

When growth of a SL producing wild-type S. bombicola strain and a SL deficient S. bombicola $\Delta c y p 52 M 1$ strain was compared on glucose and fructose concentrations of 400 or $600 \mathrm{~g} \cdot \mathrm{L}^{-1}$, no significant differences in growth curves (neither in lag, $\mu_{\max }$, or $\Delta \mathrm{OD}$ ) were noticed. Both strains showed the fastest growth (highest $\mu_{\max }$ ) on $120 \mathrm{~g} \cdot \mathrm{L}^{-1}$ sugar, with slightly higher OD values for the wild-type strain, indicating a small fitness cost of the knockout of the cyp52M1 gene. However, these small differences are no longer prevalent for higher sugar concentrations, i.e., almost no growth remaining at $600 \mathrm{~g} \cdot \mathrm{L}^{-1}$. The hypothesis that the production of SLs was selected for due to the protection of S. bombicola by SLs against high osmotic pressure can thus be rejected.

\subsection{Uptake of Hydrophobic Substrates}

SLs are classified as biosurfactants and are known for their emulsifying properties which could enhance contact with and the subsequent uptake of water-insoluble hydrophobic substrates (e.g., applied in bioremediation). The only supporting data for this hypothesis as the natural function of SLs are provided by Ito et al., mentioning a shortened lag phase on hexadecane when $0.4 \mathrm{~g} \cdot \mathrm{L}^{-1}$ wild-type SLs were added to the medium [39]. Contrary results were obtained in this study: the addition of $1 \mathrm{~g} \cdot \mathrm{L}^{-1} \mathrm{SLs}$ halted the little growth that was observed in the cultures without the SL addition (Figure S6). It is not clear if this discrepancy is caused by a different SL composition (Ito et al. claimed that monoand di-acetylated lactonic SLs were not responsible for growth stimulation) or a higher added SL concentration. If SLs indeed improve the availability of hexadecane to the cells and the latter would be toxic at a certain concentration, too high SL concentrations might be detrimental.

SLs could not induce growth on beeswax, which corresponds to the fact that Detry et al. could not isolate yeasts from surface swabs of empty wax cells [79]. However, SLs are able to increase the growth rate on rapeseed oil significantly for a SL deficient $\triangle c y p 52 M 1$ strain (but are no prerequisite for growth) and hence might confer their producing microorganism with an evolutionary benefit (Figure 4). It is not clear if this growth benefit applies to other 
hydrophobic substrates that can be catabolized by S. bombicola. This should be further investigated besides the mechanism for this enhanced growth in the presence of SLs, which is probably the promoted uptake of the hydrophobic substrate through emulsification. This enhanced uptake of substrates such as triglycerides can in turn reinforce the SL production as they can be incorporated in newly produced SLs and, as such, invigorate additional competitive advantages of SLs (as described below).

However, the presence of hydrophobic substrates does not (directly) 'induce' SL production: for growth on rapeseed oil, a SL producing wild type did not show an advantage over a non-SL producing $\triangle c y p 52 M 1$ strain when no SLs were added, and SLs could not be detected in the culture broth of the wild type at 7 days of incubation. Moreover, SLs are also synthesized when only glucose is present in the medium (see Figure S1). These observations suggest that the improved uptake of hydrophobic substrates may be a less important natural function of SLs.

\subsection{Exclusive Storage Compound}

Lastly, the catabolization of SLs by S. bombicola was also investigated. SLs disappeared from the production medium during prolonged incubation and S. bombicola is able to grow on SLs as the sole C-source and can thus effectuate catabolization of its own glycolipids in contrast to what was stated previously [66]. This catabolization consists of the action of secreted enzymes at least responsible for the ring-opening of (di-acetylated) lactonic SLs and deacetylation of acetylated acidic and lactonic SLs. These extracellular processes eventually give rise to non-acetylated acidic SLs.

This theory also holds for P. bogoriensis, as the disappearance of its branched SLs from old culture medium was already reported in 1961 [70]. In agreement with our findings, the gradual disappearance of di-acetylated SLs occurred after 3.5 days of cultivation of $P$. bogoriensis, and the simultaneous appearance of mono- and non-acetylated derivatives was observed [80]. An acetylesterase capable of performing these deacetylation reactions in P. bogoriensis was identified some years later [81].

Incubation of non-acetylated acidic SLs with the unconcentrated secretomes of S. bombicola did not lead to the formation of glucolipids and/or fatty acids, so acetylation does not seem to be a protective mechanism against attack by an extracellular glycosylhydrolase-like enzyme, which is the case for the cellobiose lipid biosurfactants produced by P. flocculosa (flocculosin): deac(et)ylation of the glycolipids at high $\mathrm{pH}$ values leads to a very fast extracellular metabolization of the deac(et)ylated derivatives [67]. This indicates that these non-acetylated acidic molecules are the end products of the extracellular catabolization of SLs by S. bombicola. They are hypothesized to be taken up again by the starved cells-which requires the presence of a SL transporter other than the MDR transporter, as this is an $\mathrm{ABC}$ transporter, and these are exclusively reported to show export activity-to be further catabolized intracellularly, explaining their disappearance of cultures cultivated on SLs as the sole carbon source. However, acetylation could still be a protective mechanism to prevent the uptake of acetylated SL derivatives, as was suggested for other types of molecules by Danchin [82].

Further catabolization of non-acetylated acidic SLs by hydrolysis of the glycosidic linkage between the two glucose moieties and between the sophorose- and fatty acid moiety was shown to happen (predominantly) intracellularly. For SL catabolization, at least a stepwise removal of the glucose molecules exists, as both glucolipids and hydroxylated fatty acids were detected in the assays. Whereas Garcia-Ochoa et al. (1996) reported the substantial release of sophorose from SLs, we did not share this observation, as sophorose was only detected in very small amounts [64]. However, the possible enzymatic cleavage of the released sophorose could lead to an underestimation of the total release of this disaccharide in the assays. For comparison: for flocculosin (cellobiose lipid), glucose was shown to be released by catabolic enzymes, whereas cellobiose release was never detected [67]. 
The major catabolic route for S. bombicola is thus suggested to consist of extracellular ring-opening and deacetylation, eventually leading to non-acetylated acidic SLs. These molecules are subsequently taken up by the starved cells and further catabolized intracellularly into glucolipids, hydroxylated fatty acids, sophorose, and glucose. This is an effective strategy to protect the extracellular storage material (especially with antimicrobial activity) against other competing microorganisms, leaving the carbon 'secured'. Complete and efficient extracellular degradation of deacetylated acidic SLs would lead to the release of glucose and fatty acids in the extracellular space, also accessible to other (competing) microorganisms.

\subsection{SLs and Their Possible Relation to Overwintering}

New ensuing questions arise: 'Which genes are responsible for the catabolization of sophorolipids in S. bombicola?' and 'Why does this yeast claim nutrients and convert them into exclusive storage compounds in an environment characterized by the prevalence of sugars?'. The first question could be solved by performing proteomic studies combined with knocking out the suspected genes. The resulting information could be very useful to improve SL production by eliminating the catabolic pathway. For the second question, we present the following theory.

S. bombicola, and in extension the whole Starmerella clade, is closely associated with a flower-bee mutualism: a natural environment abundant in sugars, found in nectar and honey $[57,83]$. However, in temperate climates, these resources evanesce in winter when flowers and pollinators disappear. Some researchers already have wondered how 'nectar yeasts' survive harsh winters: some believe the yeasts overwinter in soil and are redistributed in spring to new budding flowers through wind or crawling insects; others found them to overwinter together with the associated insect [57]. Each species possibly has its own overwintering strategy.

The nectar yeast Candida bombi, currently renamed to Starmerella bombi, was found to overwinter in the digestive tract of hibernating bumblebee queens [57,84,85]. As this yeast is closely related to $S$. bombicola (the $18 \mathrm{~S}$ rDNA genes share a $96 \%$ sequence identity), it is very reasonable that the same overwintering strategy also holds for the latter.

Moreover, several observations strengthen a close association of bumblebees and S. bombicola. Although some easily generalize the habitat of S. bombicola to 'bees and honey', Spencer et al. mentioned that despite being isolated first from nectar, they never obtained such high numbers as in bumblebee honey (Bombus ssp.) [86]. Additionally, S. bombicola was only isolated once from honey bees (A. mellifera) or its associated products [79]. These bee species differ in some remarkable ways.

First of all, sugar concentrations in the finished honey of A. mellifera are around 84\%, too high to allow the growth of yeasts. This is also reflected by the data in Figure 3: growth is already strongly repressed at $400 \mathrm{~g} \cdot \mathrm{L}^{-1}$ and almost completely ceased at $600 \mathrm{~g} \cdot \mathrm{L}^{-1}$. In addition, honey bee honey is characterized by an equal amount of glucose and fructose. In contrast, bumblebees produce, next to the 'thick' concentrated honey with $70-87 \%$ sugar, also 'thin' honey with sugar concentrations of $42-52 \%$ (from this thin honey, Spencer et al. isolated $S$. bombicola). Moreover, bumblebee honey contains much more fructose than glucose, matching the fructophilic nature of $S$. bombicola (see Table 1) $[61,87,88]$.

Lastly, and most importantly, there is a crucial difference in their behavior: honey bees actively survive winter as a colony, thereby relying on their stored honey, whereas for bumblebees, only the new queens overwinter $[89,90]$. Before wintertime, the new queens accumulate reserves of fat and glycogen. With some last honey in their crop, they leave the old nests and burrow into the soil where they hibernate. When spring arrives, they fly out seeking nectar and meanwhile inoculating new sterile flowers with yeasts. The more yeast cells that are present on the hibernated queen, the more successful they can colonize flowers and subsequently redistribute [91]. Surprisingly, the relative abundance of $S$. bombi seems to increase during hibernation, indicating an improved fitness towards other 'bumblebee yeasts' [85]. As hibernating queens are far less metabolically active, and 
the supply of sugars for the yeasts diminish, an exclusive storage of carbon and energy would be an excellent strategy to keep up cell numbers, preparing the yeast for a head start at early spring. Although no SL production is yet reported for S. bombi (which could be due to nonoptimal culture conditions), this exclusive storage compound could be sophorolipids. The same could hold for S. bombicola, known to produce excessive amounts of SLs and able to metabolize them steadily (see Figures 5 and 7) to maintain cell numbers to a constant level for over 100 days, which is as long as a winter.

\section{Conclusions}

To summarize, it could not be confirmed that sophorolipids (SLs) protect its producing yeast $S$. bombicola against high osmotic pressure caused by the prevalence of sugars in the yeasts' natural habitat. On the other hand, wild-type SLs do exhibit antimicrobial activity against a broad range of microorganisms. Furthermore, S. bombicola can catabolize its own previously produced SLs: deacetylation and ring-opening occur extracellularly, resulting in non-acetylated acidic SLs which are imported and further degraded intracellularly. On rapeseed oil, increased growth rates were noticed when SLs were added to a $\Delta c y p 52 M 1$ S. bombicola culture. Further research is needed to confirm the improved uptake through emulsification as the underlying mechanism. However, some observations indicate a less important physiological function of SLs in the growth on hydrophobic substrates. Nevertheless, an improved uptake of the hydrophobic substrate can boost SL production and hence invigorate the natural functions mentioned above.

Based on the results mentioned above, we proclaim that the natural function of SLs in S. bombicola is the build-up of an exclusive, extracellular storage compound with inherent antimicrobial activity that can be utilized under starvation conditions. In this way, S. bombicola can very efficiently compete for the available carbon and energy sources with other microorganisms populating its habitat; valuable and easily degradable carbon sources, such as sugars and/or fatty acids, are claimed by converting them into more inert compounds: sophorolipids.

Supplementary Materials: The following are available online at https:/ /www.mdpi.com/article/10 .3390/jof7110917/s1. Figure S1: UPLC-ELSD chromatograms of the culture broth of the S. bombicola wild-type strain and the S. bombicola $\triangle c y p 52 M 1$ strain grown on glucose and fructose. Figure S2: Light microscopic observations of a $S$. bombicola wild-type and a $\triangle$ cyp52M1 strain that were cultivated on 20, 120, and $400 \mathrm{~g} \cdot \mathrm{L}^{-1}$ fructose or glucose. Figure S3: Growth curves of a S. bombicola wild-type and $\triangle c y p 52 M 1$ strain on medium with glucose or sucrose. Figure S4: TLC analysis of cell culture broths of a S. bombicola wild-type and a $\triangle$ cyp52M1 strain on medium with rapeseed oil as the sole carbon source. Figure S5: Growth curves of a S. bombicola wild-type and $\triangle c y p 52 M 1$ strain on medium with rapeseed oil as sole carbon source. Figure S6: Scatterplots of the estimated growth parameters of a sophorolipid deficient S. bombicola $\triangle$ cyp52M1 strain and a sophorolipid producing S. bombicola wild-type strain on hexadecane. Table S1: Influence of $\mathrm{pH}$ on the solubility $\left(\mathrm{g} \cdot \mathrm{L}^{-1}\right)$ of different forms of SLs. Figure S7: HPLC-ELSD chromatograms of samples from a S. bombicola culture on SL medium with di-acetylated lactonic SLs as the sole carbon source. Table S2: Identification of the major peaks detected in Figure S7 after HPLC-MS analysis. Figure S8: HPLC-ELSD chromatograms of samples from an extracellular activity assay with a S. bombicola $\Delta$ sble strain (unconcentrated secretome) incubated with di-acetylated lactonic SLs. Figure S9: HPLC-ELSD chromatograms of an enzyme assay of the wild-type lysate and di-acetylated lactonic SLs. Table S3: Identification of the major peaks detected in Figure S9 after HPLC-MS analysis. 
Author Contributions: Conceptualization, V.D.C. and S.L.K.W.R.; Methodology, V.D.C., S.L.K.W.R. and M.G.C.; Investigation, V.D.C., S.L.K.W.R. and M.G.C.; Formal Analysis, V.D.C., S.L.K.W.R. and M.G.C.; Software, V.D.C.; Visualization, V.D.C. and S.L.K.W.R.; Validation, S.L.K.W.R., S.L.D.M., V.D.C. and M.G.C.; Data Curation, S.L.K.W.R., V.D.C. and S.L.D.M.; Writing-Original Draft Preparation, V.D.C., M.G.C. and S.L.K.W.R.; Writing-Review and Editing, V.D.C., S.L.D.M. and S.L.K.W.R.; Supervision, S.L.D.M., S.L.K.W.R. and W.K.S.; Project Administration, S.L.K.W.R., S.L.D.M. and W.K.S.; Funding Acquisition, S.L.K.W.R., S.L.D.M. and W.K.S.; Resources, W.K.S. All authors have read and agreed to the published version of the manuscript.

Funding: This research was funded by the Research Foundation-Flanders with grant number 1S58817N (SB PhD fellow); by the Institute for the Promotion of Innovation through Science and Technology in Flanders with grant number IWT80050 (IWT-Vlaanderen, Biosurf); by the European FP7 project with grant number 289219 (Biosurfing); and by Flanders Innovation \& Entrepreneurship with grant number HBC.2017.0704 (Vlaio-VIS Intercluster, Applisurf).

Institutional Review Board Statement: Not applicable.

Informed Consent Statement: Not applicable.

Data Availability Statement: The data presented in this study are available in the results section or in the Supplementary Material of this article.

Acknowledgments: A special thanks goes to Maarten De Mol for his boundless advice in our never-ending discussions.

Conflicts of Interest: The authors declare no conflict of interest.

\section{References}

1. De Oliveira, M.R.; Camilios-Neto, D.; Baldo, C.; Magri, A.; Pedrine, M.A.; Celligoi, C. Biosynthesis and Production of Sophorolipids. Int. J. Sci. Technol. Res. 2014, 3, 133-146.

2. De Graeve, M.; de Maeseneire, S.L.; Roelants, S.L.K.W.; Soetaert, W. Starmerella bombicola, an industrially relevant, yet fundamentally underexplored yeast. FEMS Yeast Res. 2018, 18, foy072. [CrossRef]

3. Asmer, H.-J.; Lang, S.; Wagner, F.; Wray, V. Microbial production, structure elucidation and bioconversion of sophorose lipids. J. Am. Oil Chem. Soc. 1988, 65, 1460-1466. [CrossRef]

4. Cavalero, D.A.; Cooper, D.G. The effect of medium composition on the structure and physical state of sophorolipids produced by Candida bombicola ATCC 22214. J. Biotechnol. 2003, 103, 31-41. [CrossRef]

5. Kurtzman, C.P.; Price, N.P.J.; Ray, K.J.; Kuo, T.-M. Production of sophorolipid biosurfactants by multiple species of the Starmerella (Candida) bombicola yeast clade. FEMS Microbiol. Lett. 2010, 311, 140-146. [CrossRef] [PubMed]

6. Davila, A.M.; Marchal, R.; Monin, N.; Vandecasteele, J.P. Identification and determination of individual sophorolipids in fermentation products by gradient elution high-performance liquid chromatography with evaporative light-scattering detection. J. Chromatogr. A 1993, 648, 139-149. [CrossRef]

7. Chen, M.; Dong, C.; Penfold, J.; Thomas, R.K.; Smyth, T.J.P.; Perfumo, A.; Marchant, R.; Banat, I.M.; Stevenson, P.; Parry, A.; et al. Adsorption of Sophorolipid Biosurfactants on Their Own and Mixed with Sodium Dodecyl Benzene Sulfonate, at the Air/Water Interface. Langmuir 2011, 27, 8854-8866. [CrossRef] [PubMed]

8. Imura, T.; Kawamura, D.; Taira, T.; Morita, T.; Fukuoka, T.; Aburai, K.; Sakai, H.; Abe, M.; Kitamoto, D. Monolayer Behavior of Binary Systems of Lactonic and Acidic Forms of Sophorolipids: Thermodynamic Analyses of Langmuir Monolayers and AFM Study of Langmuir-Blodgett Monolayers. J. Oleo Sci. 2014, 63, 67-73. [CrossRef]

9. Tulloch, A.P.; Hill, A.; Spencer, J.F.T. Structure and reactions of lactonic and acidic sophorosides of 17-hydroxyoctadecanoic acid. Can. J. Chem. 1968, 46, 3337-3351. [CrossRef]

10. $\mathrm{Hu}, \mathrm{Y}$; Ju, L.K. Sophorolipid production from different lipid precursors observed with LC-MS. Enzyme Microb. Technol. 2001, 29, 593-601. [CrossRef]

11. Hall, P.J.; Haverkamp, J.; van Kralingen, C.G.; Schmidt, M. Synergistic Dual-Surfactant Detergent Composition Containing Sophoroselipid 1993. U.S. Patent 5,417,879, 23 May 1995.

12. Furuta, T.; Igarashi, K.; Hirata, Y. Low-Foaming Detergent Compositions 2002. U.S. Patent Application Publication US20040171512A1, 2 September 2004.

13. Shete, A.M.; Wadhawa, G.; Banat, I.M.; Chopade, B.A. Mapping of patents on bioemulsifier and biosurfactant: A review. J. Sci. Ind. Res. 2006, 65, 91-115.

14. Roberto de Oliveira, M.; Magri, A.; Baldo, C.; Camilios-Neto, D.; Minucelli, T.; Pedrine, M.A.; Celligoi, C. Review: Sophorolipids A Promising Biosurfactant and it's Applications. Int. J. Adv. Biotechnol. Res. 2015, 6, 161-174.

15. Lydon, H.L.; Baccile, N.; Callaghan, B.; Marchant, R.; Mitchell, C.A.; Banat, I.M. Adjuvant antibiotic activity of acidic sophorolipids with potential for facilitating wound healing. Antimicrob. Agents Chemother. 2017, 61, e02547-16. [CrossRef] 
16. Van Renterghem, L.; Roelants, S.L.K.W.; Baccile, N.; Uyttersprot, K.; Taelman, M.C.; Everaert, B.; Mincke, S.; Ledegen, S.; Debrouwer, S.; Scholtens, K.; et al. From lab to market: An integrated bioprocess design approach for new-to-nature biosurfactants produced by Starmerella bombicola. Biotechnol. Bioeng. 2018, 115, 1195-1206. [CrossRef]

17. Arab, F.; Mulligan, C.N. An eco-friendly method for heavy metal removal from mine tailings. Environ. Sci. Pollut. Res. 2018, 25, 16202-16216. [CrossRef]

18. Lodens, S.; de Graeve, M.; Roelants, S.L.K.W.; de Maeseneire, S.L.; Soetaert, W. Transformation of an exotic yeast species into a platform organism: A case study for engineering glycolipid production in the yeast starmerella bombicola. In Synthetic Biology. Methods in Molecular Biology; Braman, J., Ed.; Humana Press: New York, NY, USA, 2018; Volume 1772, pp. 95-123.

19. Van Bogaert, I.N.A.; Buyst, D.; Martins, J.C.; Roelants, S.L.K.W.; Soetaert, W.K. Synthesis of bolaform biosurfactants by an engineered Starmerella bombicola yeast. Biotechnol. Bioeng. 2016, 113, 2644-2651. [CrossRef] [PubMed]

20. Saerens, K.M.J.; Zhang, J.; Saey, L.; van Bogaert, I.N.A.; Soetaert, W. Cloning and functional characterization of the UDPglucosyltransferase UgtB1 involved in sophorolipid production by Candida bombicola and creation of a glucolipid-producing yeast strain. Yeast 2011, 28, 279-292. [CrossRef] [PubMed]

21. Lodens, S.; Roelants, S.L.K.W.; Ciesielska, K.; Geys, R.; Derynck, E.; Maes, K.; Pattyn, F.; van Renterghem, L.; Mottet, L.; Dierickx, S.; et al. Unraveling and resolving inefficient glucolipid biosurfactants production through quantitative multiomics analyses of Starmerella bombicola strains. Biotechnol. Bioeng. 2020, 117, 453-465. [CrossRef]

22. Spencer, J.F.; Gorin, P.A.; Tulloch, A.P. Torulopsis bombicola sp.n. Antonie Van Leeuwenhoek 1970, 36, 129-133. [CrossRef]

23. Yarrow, D.; Meyer, S.A. Proposal for amendment of the diagnosis of the genus Candida Berkhout nom. cons. Int. J. Syst. Bacteriol. 1978, 28, 611-615. [CrossRef]

24. McNeill, J.; Barrie, F.R.; Buck, W.R.; Demoulin, V.; Greuter, W.; Hawksworths, D.L.; Herendeen, P.S.; Knapp, S.; Marhold, K.; Prado, J.; et al. International Code of Nomenclature for Algae, Fungi and Plants (Melbourne Code) Adopted by the Eighteenth International Botanical Congress Melbourne, Australia, July 2011; Koeltz Scientific Books: Königstein, Germany, 2012; Volume 154, ISBN 9783874294256.

25. Crane, E. Honey from honeybees and other insects. Ethol. Ecol. Evol. 1991, 3, 100-105. [CrossRef]

26. Chalcoff, V.R.; Aizen, M.A.; Galetto, L. Nectar concentration and composition of 26 species from the temperate forest of South America. Ann. Bot. 2006, 97, 413-421. [CrossRef]

27. Aleklett, K.; Hart, M.; Shade, A. The microbial ecology of flowers: An emerging frontier in phyllosphere research1. Botany 2014, 92, 253-266. [CrossRef]

28. Fratini, F.; Cilia, G.; Turchi, B.; Felicioli, A. Beeswax: A minireview of its antimicrobial activity and its application in medicine. Asian Pac. J. Trop. Med. 2016, 9, 839-843. [CrossRef]

29. Hammond, E.W. Vegetable Oils I Types and Properties. In Encyclopedia of Food Sciences and Nutrition; Academic Press: Cambridge, MA, USA, 2003; pp. 5899-5904.

30. Davila, A.M.; Marchal, R.; Vandecasteele, J.P. Sophorose lipid fermentation with differentiated substrate supply for growth and production phases. Appl. Microbiol. Biotechnol. 1997, 47, 496-501. [CrossRef]

31. Ito, S.; Kinta, M.; Inoue, S. Growth of yeasts on n-alkanes: Inhibition by a lactonic sophorolipid produced by Torulopsis bombicola. Agric. Biol. Chem. 1980, 44, 2221-2223. [CrossRef]

32. Tran, H.G. Improving the Glycosylation Activity of Cellobiose/Cellodextrin Phosphorylase through Enzyme Engineering; Ghent University: Ghent, Belgium, 2012.

33. Gross, R.A.; Shah, V. Antifungal Properties of Various Forms of Sophorolipids 2003. World Intellectual Property Organization. WO2004044216A1, 27 May 2004.

34. Díaz de Rienzo, M.A.; Stevenson, P.; Marchant, R.; Banat, I.M. Antibacterial properties of biosurfactants against selected Gram-positive and -negative bacteria. FEMS Microbiol. Lett. 2016, 363, fnv224. [CrossRef] [PubMed]

35. Kim, K.; Yoo, D.; Kim, Y.; Lee, B.-S.; Shin, D.; Kim, E.K. Characteristics of sophorolipid as an antimicrobial agent. J. Microbiol. Biotechnol. 2002, 12, 235-241.

36. Haque, F.; Alfatah, M.; Ganesan, K.; Bhattacharyya, M.S. Inhibitory Effect of Sophorolipid on Candida albicans Biofilm Formation and Hyphal Growth. Sci. Rep. 2016, 6, 1-11. [CrossRef]

37. Hipólito, A.; Alves da Silva, R.A.; de Oliveira Caretta, T.; Itakura Silveira, V.A.; Amador, I.R.; Panagio, L.A.; Borsato, D.; Pedrine Colabone Celligoi, M.A. Evaluation of the antifungal activity of sophorolipids from Starmerella bombicola against food spoilage fungi. Biocatal. Agric. Biotechnol. 2020, 29, 101797. [CrossRef]

38. Hommel, R.K.; Weber, L.; Weiss, A.; Himmelreich, U.; Rilke, O.; Kleber, H.P. Production of sophorose lipid by Candida (Torulopsis) apicola grown on glucose. J. Biotechnol. 1994, 33, 147-155. [CrossRef]

39. Ito, S.; Inoue, S. Sophorolipids from Torulopsis bombicola: Possible relation to alkane uptake. Appl. Environ. Microbiol. 1982, 43, 1278-1283. [CrossRef]

40. Hommel, R.K. Formation and physiological role of biosurfactants produced by hydrocarbon-utilizing microorganismsBiosurfactants in hydrocarbon utilization. Biodegradation 1990, 1, 107-119. [CrossRef]

41. Linton, J.D. Metabolite production and growth efficiency. Antonie Van Leeuwenhoek 1991, 60, 293-311. [CrossRef] [PubMed]

42. Dierickx, S.; Castelein, M.; Remmery, J.; De Clercq, V.; Lodens, S.; Baccile, N.; De Maeseneire, S.L.; Roelants, S.L.K.W.; Soetaert, W.K. From bumblebee to bioeconomy: Recent developments and perspectives for sophorolipid biosynthesis. Biotechnol. Adv. 2021, 107788. [CrossRef] [PubMed] 
43. CLSI. Methods for Dilution Antimicrobial Susceptibility Tests for Bacteria That Grow Aerobically (CLSI Standard M07), 11th ed.; Clinical and Laboratory Standards Institute: Wayne, PA, USA, 2018.

44. Balouiri, M.; Sadiki, M.; Ibnsouda, S.K. Methods for in vitro evaluating antimicrobial activity: A review. J. Pharm. Anal. 2016, 6, 71-79. [CrossRef] [PubMed]

45. Elshikh, M.; Moya-Ramírez, I.; Moens, H.; Roelants, S.; Soetaert, W.; Marchant, R.; Banat, I.M. Rhamnolipids and lactonic sophorolipids: Natural antimicrobial surfactants for oral hygiene. J. Appl. Microbiol. 2017, 123, 1111-1123. [CrossRef]

46. Elshikh, M.; Ahmed, S.; Funston, S.; Dunlop, P.; McGaw, M.; Marchant, R.; Banat, I.M. Resazurin-based 96-well plate microdilution method for the determination of minimum inhibitory concentration of biosurfactants. Biotechnol. Lett. 2016, 38, 1015-1019. [CrossRef] [PubMed]

47. Van Bogaert, I.N.A.; Holvoet, K.; Roelants, S.L.K.W.; Li, B.; Lin, Y.C.; van de Peer, Y.; Soetaert, W. The biosynthetic gene cluster for sophorolipids: A biotechnological interesting biosurfactant produced by Starmerella bombicola. Mol. Microbiol. 2013, 88, 501-509. [CrossRef]

48. Ciesielska, K.; van Bogaert, I.N.; Chevineau, S.; Li, B.; Groeneboer, S.; Soetaert, W.; van de Peer, Y.; Devreese, B. Exoproteome analysis of Starmerella bombicola results in the discovery of an esterase required for lactonization of sophorolipids. J. Proteomics 2014, 98, 159-174. [CrossRef]

49. Roelants, S.L.K.W.; Ciesielska, K.; de Maeseneire, S.L.; Moens, H.; Everaert, B.; Verweire, S.; Denon, Q.; Vanlerberghe, B.; van Bogaert, I.N.A.; van der Meeren, P.; et al. Towards the Industrialization of New Biosurfactants: Biotechnological Opportunities for the Lactone Esterase Gene From Starmerella bombicola. Biotechnol. Bioeng. 2016, 113, 550-559. [CrossRef]

50. Lang, S.; Brakemeier, A.; Heckmann, R.; Spockner, S.; Rau, U. Production of native and modified sophorose lipids. Chim. Oggi-Chem. Today 2000, 18, 76-79.

51. Saerens, K.M.J.; Roelants, S.L.K.W.; van Bogaert, I.N.A.; Soetaert, W. Identification of the UDP-glucosyltransferase gene UGTA1, responsible for the first glucosylation step in the sophorolipid biosynthetic pathway of Candida bombicola ATCC 22214. FEMS Yeast Res. 2011, 11, 123-132. [CrossRef]

52. Pozo, M.I.; Lievens, B.; Jacquemyn, H. Impact of Microorganisms on Nectar Chemistry, Pollinator Attraction and Plant Fitness. In Nectar: Production, Chemical Composition and Benefits to Animals and Plants; Nova Science Publishers Inc.: New York, NY, USA, 2015; pp. 1-45.

53. Vit, P.; Roubik, D.W.; Pedro, S.R.M. Pot-Honey: A Legacy of Stingless Bees; Springer: New York, NY, USA, $2012 ;$ ISBN 9781461449607.

54. De Vega, C.; Albaladejo, R.G.; Guzmán, B.; Steenhuisen, S.; Johnson, S.D.; Herrera, C.M.; Lachance, M. Flowers as a reservoir of yeast diversity: Description of Wickerhamiella nectarea f.a. sp. nov., and Wickerhamiella natalensis f.a. sp. nov. from South African flowers and pollinators, and transfer of related Candida species to the genus Wickerhamiella as new combinations. FEMS Yeast Res. 2017, 17, fox054. [CrossRef]

55. Sandhu, D.K.; Waraich, M.K. Yeasts Associated with Pollinating Bees and Flower Nectar. Microb. Ecol. 1985, 11, 51-58. [CrossRef]

56. Praet, J.; Parmentier, A.; Schmid-Hempel, R.; Meeus, I.; Smagghe, G.; Vandamme, P. Large-scale cultivation of the bumblebee gut microbiota reveals an underestimated bacterial species diversity capable of pathogen inhibition. Environ. Microbiol. 2018, 20, 214-227. [CrossRef]

57. Brysch-Herzberg, M. Ecology of yeasts in plant-bumblebee mutualism in Central Europe. FEMS Microbiol. Ecol. 2004, 50, 87-100. [CrossRef]

58. Endo, A. Fructophilic lactic acid bacteria inhabit fructose-rich niches in nature. Microb. Ecol. Health Dis. 2012, 23, 18563. [CrossRef]

59. Babazadeh, R.; Lahtvee, P.J.; Adiels, C.B.; Goksör, M.; Nielsen, J.B.; Hohmann, S. The yeast osmostress response is carbon source dependent. Sci. Rep. 2017, 7, 1-11. [CrossRef]

60. Sleator, R.D.; Hill, C. Bacterial osmoadaptation: The role of osmolytes in bacterial stress and virulence. FEMS Microbiol. Rev. 2002, 26, 49-71. [CrossRef] [PubMed]

61. Gonçalves, C.; Wisecaver, J.H.; Kominek, J.; Salema Oom, M.; Leandro, M.J.; Shen, X.X.; Opulente, D.A.; Zhou, X.; Peris, D.; Kurtzman, C.P.; et al. Evidence for loss and reacquisition of alcoholic fermentation in a fructophilic yeast lineage. elife $2018,7$. [CrossRef] [PubMed]

62. Hommel, R.K.; Huse, K. Regulation of sophorose lipid production by Candida (Torulopsis) apicola. Biotechnol. Lett. 1993, 15, 853-858. [CrossRef]

63. Jules, M.; Beltran, G.; François, J.; Parrou, J.L. New insights into trehalose metabolism by Saccharomyces cerevisiae: NTH2 encodes a functional cytosolic trehalase, and deletion of TPS1 reveals Ath1p-dependent trehalose mobilization. Appl. Environ. Microbiol. 2008, 74, 605-614. [CrossRef]

64. Garcia-Ochoa, F.; Casas, J.A. Process for the Production of Sophorose by Candida Bombicola 1996. Spanish Patent ES2103688A1, 16 September 1997.

65. Hu, Y. Production, Purification and Enzymatic Polymerization of Sophorolipids; University of Akron: Akron, OH, USA, 2000.

66. Lo, C.M.; Ju, L.K. Sophorolipids-induced cellulase production in cocultures of Hypocrea jecorina Rut C 30 and Candida bombicola. Enzyme Microb. Technol. 2009, 44, 107-111. [CrossRef]

67. Mimee, B.; Labbé, C.; Bélanger, R.R. Catabolism of flocculosin, an antimicrobial metabolite produced by Pseudozyma flocculosa. Glycobiology 2009, 19, 995-1001. [CrossRef]

68. Abdel-Mawgoud, A.M.; Aboulwafa, M.M.; Hassouna, N.A.H. Optimization of surfactin production by bacillus subtilis isolate BS5. Appl. Biochem. Biotechnol. 2008, 150, 305-325. [CrossRef] [PubMed] 
69. Kitamoto, D.; Nakane, T.; Nakao, N.; Nakahara, T.; Tabuchi, T. Intracellular accumulation of mannosylerythritol lipids as storage materials by Candida antarctica. Appl. Microbiol. Biotechnol. 1992, 36, 768-772. [CrossRef]

70. Deinema, M. Intra- and Extra-Cellular Lipid Production by Yeasts; University of Wageningen: Wageningen, The Netherlands, 1961.

71. Stüwer, O.; Hommel, R.; Haferburg, D.; Kleber, H.P. Production of crystalline surface-active glycolipids by a strain of torulopsis apicola. J. Biotechnol. 1987, 6, 259-269. [CrossRef]

72. Albrecht, A.; Rau, U.; Wagner, F. Initial steps of sophoroselipid biosynthesis by Candida bombicola ATCC 22214 grown on glucose. Appl. Microbiol. Biotechnol. 1996, 46, 67-73. [CrossRef]

73. Saerens, K.M.J.; Saey, L.; Soetaert, W. One-step production of unacetylated sophorolipids by an acetyltransferase negative Candida bombicola. Biotechnol. Bioeng. 2011, 108, 2923-2931. [CrossRef]

74. Joshi-Navare, K.; Khanvilkar, P.; Prabhune, A. Jatropha Oil Derived Sophorolipids: Production and Characterization as Laundry Detergent Additive. Biochem. Res. Int. 2013, 2013, 1-11. [CrossRef]

75. Luong, N.; Hoa, H.; Loan, L.Q.; Eun-ki, K.; Ha, T.T.; Duy, N.D.; Khanh, H.Q.; Dung, N.H. Production and characterization of sophorolipids produced by Candida bombicola using sugarcane molasses and coconut oil. Asia-Pacific J. Sci. Technol. 2017, 22, 1-8. [CrossRef]

76. Akemi, V.; Silveira, I.; Urzedo, C.A.; Freitas, Q.; Pedrine, M.A.; Celligoi, C. Antimicrobial applications of sophorolipid from Candida bombicola: A promising alternative to conventional drugs. J. Appl. Biol. Biotechnol. 2018, 6, 87-90. [CrossRef]

77. Dengle-Pulate, V.; Chandorkar, P.; Bhagwat, S.; Prabhune, A.A. Antimicrobial and SEM studies of sophorolipids synthesized using lauryl alcohol. J. Surfactants Deterg. 2014, 17, 543-552. [CrossRef]

78. Pohl, A.; Lübke-Becker, A.; Heuwieser, W. Minimum inhibitory concentrations of frequently used antibiotics against Escherichia coli and Trueperella pyogenes isolated from uteri of postpartum dairy cows. J. Dairy Sci. 2018, 101, 1355-1364. [CrossRef]

79. Detry, R.; Simon-Delso, N.; Bruneau, E.; Daniel, H.-M. Specialisation of Yeast Genera in Different Phases of Bee Bread Maturation. Microorganisms 2020, 8, 1789. [CrossRef] [PubMed]

80. Esders, T.W.; Light, R.J. Characterization and in vivo production of three glycolipids from Candida Bogoriensis: 13glucopyranosylglucopyranosyloxydocosanoic acid and its mono- and diacetylated derivatives. J. Lipid Res. 1972, $13,663-671$. [CrossRef]

81. Bucholtz, M.L.; Light, R.J. Hydrolysis of 13-sophorosyloxydocosanoic acid esters by acetyl- and carboxylesterases isolated from Candida bogoriensis. J. Biol. Chem. 1976, 251, 431-437. [CrossRef]

82. Danchin, A. Cells need safety valves. BioEssays 2009, 31, 769-773. [CrossRef]

83. Rosa, C.A.; Lachance, M.A.; Silva, J.O.C.; Teixeira, A.C.P.; Marini, M.M.; Antonini, Y.; Martins, R.P. Yeast communities associated with stingless bees. FEMS Yeast Res. 2003, 4, 271-275. [CrossRef]

84. Santos, A.R.O.; Leon, M.P.; Barros, K.O.; Freitas, L.F.D.; Hughes, A.F.S.; Morais, P.B.; Lachance, M.A.; Rosa, C.A. Starmerella camargoi f.A., sp. nov., starmerella ilheusensis f.a., sp. nov., Starmerella litoralis f.a., sp. nov., starmerella opuntiae f.a., sp. nov., Starmerella roubikii f.a., sp. nov. and Starmerella vitae f.a., sp. nov., isolated from flowers and bee. Int. J. Syst. Evol. Microbiol. 2018, 68, 1333-1343. [CrossRef]

85. Pozo, M.I.; Bartlewicz, J.; van Oystaeyen, A.; Benavente, A.; van Kemenade, G.; Wäckers, F.; Jacquemyn, H. Surviving in the absence of flowers: Do nectar yeasts rely on overwintering bumblebee queens to complete their annual life cycle? FEMS Microbiol. Ecol. 2018, 94, fiy196. [CrossRef]

86. Spencer, J.F.T.; Spencer, D.M. Ecology: Where Yeasts Live. In Yeasts in Natural and Artificial Habitats; Springer: Berlin/Heidelberg, Germany, 1997; pp. 33-58.

87. Spencer, J.F.T.; Gorin, P.A.J.; Hobbs, G.A.; Cooke, D.A. Yeasts isolated from bumblebee honey from Western Canada: Identification with the aid of proton magnetic resonance spectra of their mannose-containing polysaccharides. Can. J. Microbiol. 1970, 16, 117-119. [CrossRef]

88. Crane, E. Bee Products. Bee World 1972, 53, 38-39. [CrossRef]

89. Johansson, T.S.K.; Johansson, M.P. The Honeybee Colony in Winter. Bee World 1979, 60, 155-170. [CrossRef]

90. Alford, D.V. A Study of the Hibernation of Bumblebees (Hymenoptera:Bombidae) in Southern England. J. Anim. Ecol. 1969, 38, 149. [CrossRef]

91. Klaps, J.; Lievens, B.; Álvarez-Pérez, S. Towards a better understanding of the role of nectar-inhabiting yeasts in plant-animal interactions. Fungal Biol. Biotechnol. 2020, 7, 1. [CrossRef] [PubMed] 